U.S. DEPARTMENT OF THE INTERIOR

GEOLOGICAL SURVEY

\title{
DETECTION OF TUNNELS BY TRANSIENT ELECTROMAGNETIC
}

SUBSURFACE IMAGING

by

Bryan A. James

Open-File Report $88-218$

1988

Any use of trade names in this report is for descriptive purposes only and does not imply endorsement by the U.S. Geological Survey. This report is preliminary and has not been reviewed for conformity with U.S. Geological Survey editorial standards. 
This report presents the concept and initial theoretical testing of transient electromagnetic (TEM) imaging - a new method of TEM processing to directly produce a two-dimensional (2-D) subsurface resistivity image - for application to tunnel detection. The imaging method utilizes the fact that all of the induced subsurface current distribution variably contributes to the measured magnetic field at all measurement locations and at all times. These contributions may be calculated from subsurface current densities for any resistivity structure, using Biot-Savart's Law. These calculated contributions (a function of subsurface position, receiver position and time) are used as weighting coefficients to extract fractions of measured magnetic field values reflecting the influence of structure within the earth unaccounted for in the assumed earth model. Summing all of these fractions for each subsurface element, normalizing by a similar sum of synthetic data for the assumed earth, and multiplying by the assumed resistivity of the element, yields an estimate of the actual resistivity of that element. Contouring the resistivity estimates for all subsurface elements provides an apparent image of the subsurface resistivity distribution.

At present there is no known way to automatically generate accurate pictures of the time-varying current density within the earth; an assumed earth model and calculation of the current density function for that model are necessary. These calculations are easily performed only for layered earth models. Practical implementation, therefore, requires an estimate of a background layered structure for the area of interest. The imaging method then yields an estimate of a 2-D or 3-D resistivity structure superimposed on the background layered structure. 
A prototype TEM imaging algorithm has been developed for the 2-D tunnel detection application and is tailored to using surface sources on a profile with profiles of receivers down boreholes. A theoretical feasibility study addresses the aspects of: tunnel with conductive shell vs. tunnel with no conductive she1l, uniform halfspace host vs. two-layer host, and loop source vs. grounded-wire source.

The study demonstrates that the TEM imaging concept is successful at producing a "fuzzy", but reasonable, estimate of the 2-D resistivity structure. Also clearly demonstrated is the need for a good estimate of the layered parameters of the host rock and use of appropriate weighting coefficients for that layered mode1; otherwise the method's resolution is very poor.

Most electromagnetic methods are much more sensitive to conductive structures than resistive structures. Consequently, as expected, this TEM imaging procedure does very well at providing a clear image only of a tunnel surrounded by a conductive shell; with no conductive shell a tunnel (a purely resistive anomaly) would likely need to be within two tunnel diameters of a borehole to be clearly recognized.

There is room for this algorithm to be improved. The most limiting problem is the need for an estimate of a proper background layered model and calculations of the current distribution for that model. Furthermore, the influence of geologic noise must be assessed to establish limits, capabilities and the appropriate role of the method in actual use. It is likely that modifications will be introduced to accommodate the vagaries of real data; a field test should be performed in the near future to assist this development. 


\section{PREFACE}

The work described in this report was funded by the U.S. Army Corps of Engineers, Waterways Experiment Station (WES), Vicksburg, Mississippi, under contract number WESAM-87-23. Dr. Dwain K. Butler was point of contact for WES. The work was performed during the period of January through September, 1987. 
ACKNOWLEDGMENT

The author would like to thank Walt Anderson of the U.S. Geological Survey for his efforts to support this work. He performed a substantial amount of work in several phases of the research. Also, and at least as important, were his moral support and friendship. 


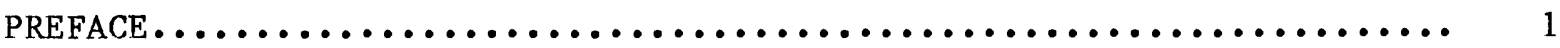

ACKNOWLEDGMENT.................................... 2

PART I: $\quad$ INTRODUCTION.............................. 4

Ba ckground.................................. 4

Objectives.................................. 5

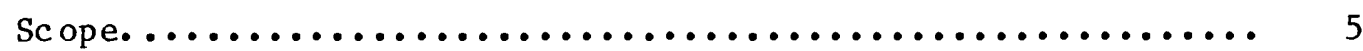

PART II: ATTRIBUTES OF TEM METHODS..................... 6

PART III: THE CONCEPT OF TRANSIENT ELECTROMAGNETIC IMAGING......... 9

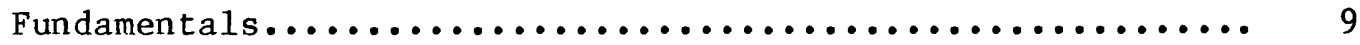

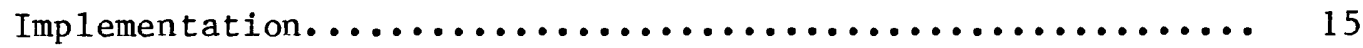

PART IV: TESTING OF THE TEM IMAGING ALGORITHM FOR TUNNEL DETECTION... 18

Tunne1 in Uniform Halfspace - Loop Source.............. 18

Effect of source-borehole separation and survey design... 18

Tunne1 with conductive shell mode1................ 21

Tunnel with no conductive shell model.............. 29

Tunnel in Uniform Halfspace - Grounded Wire Source........ 29

Tunne1 beneath Conductive Overburden - Loop Source........ 32

Discussion. .................................. 40

PART V: SUMMARY, CONCLUSIONS AND RECOMMENDATIONS............. 41

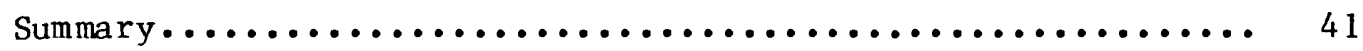

Conclusions.................................. 42

Recommendations............................. 43

REFERENCES....................................... 44 


\section{DETECTION OF TUNNELS BY TRANSIENT ELECTROMAGNETIC}

SUBSURFACE IMAGING

\section{PART I: INTRODUCTION}

\section{Background}

The detection of tunnels by any geophysical method is a difficult task. Electromagnetic (EM) methods are no exception. Furthermore, EM methods have been limited by a lack of effective and efficient interpretation capability for non-layered structure other than the special case of highly conductive orebodies (Barnett, 1984; Dyck and West, 1984). The detection of general two-dimensional structures, including tunnels, with EM methods realistically requires development of new approaches of data acquisition and interpretation.

Traditional interpretative approaches utilize analytic solutions for horizontally layered properties of the earth. These approaches are based on one-dimensional (1-D) resistivity variations and fundamentally inappropriate for mapping of two-dimensional (2-D) structures such as tunnels. However, within limits, some useful information concerning non-layered structures can be obtained with 1-D interpretation (Newman, et a1., 1987). In the author's opinion, new interpretative approaches for mapping of non-layered structures must somehow utilize the actual spatially complicated structure of the electromagnetic field (see, for example, Oristaglio and Hohmann, 1984).

Furthermore, the desired 2-D interpretation capability should not be tied directly to 2-D forward modeling capability. Computational time and the nonuniqueness properties of the EM fields are prohibitive barriers to a procedure based 2-D modeling capability. A desired goal is a two-dimensional 'image' of the subsurface that is generated directly from the recorded data 
(with as little assumption about the subsurface as possible) and is a reasonable representation of the geoelectrical structure. Such an imaging procedure has been conceived utilizing transient electromagnetic (TEM) fields. The idea of imaging with EM data is of interest to a growing number of researchers in EM methodology. It is a tool needed to assist in solving increasingly more difficult interpretation problems. Preliminary efforts have been described by Dines and Lytle (1981), Macnae and Lamontagne (1987), Lee et a1. (1987), Nekut (1987), and Levy et al. (1988).

\section{Objectives}

The objectives of the current research effort are:

(a) To develop a working TEM imaging algorithm,

(b) to implement this algorithm for the tunnel detection problem,

(c) to theoretically study the applicability of TEM imaging for tunnel detection, and

(d) to define field procedures for implementation and testing of TEM imaging on real data.

\section{Scope}

This report includes the following:

(a) A brief review of the attributes of TEM methods.

(b) A presentation of the TEM imaging concept and an algorithm for its implementation.

(c) Results of theoretical testing of the TEM imaging algorithm for tunnels in a simple layered host rock.

(d) Conclusions and recommendations regarding the TEM imaging procedure in general and its utility in tunnel detection work in particular. 
PART II: ATTRIBUTES OF TEM METHODS

TEM methods generally fall into two classes - sounding of the earth and mapping of confined conductors. Only a brief synopsis will be presented here; an excellent review of TEM methods can be found in Nabighian (1984). Figure 1 presents cartoons of key features of TEM methods for both applications. Primary features in Figure 1 are keyed to the following description. Consider the sounding application first (Figure la). Current is induced in the earth by truncation of a steady current in a loop (or grounded wire) (A). These induced currents (B) diffuse into the subsurface with increasing time in a pattern dictated by the resistivity structure (C). The diffusing currents create a time-varying magnetic field (D) which is measured at some position (E) with a magnetometer or with a coil which measures the time-rate-of-change of the magnetic field. These measurements (F) are then inverted, essentially by an iterative curve matching process comparing forward calculations with the observed data, into a layered earth model which provides the closest least-squares fit to the data. This model is presumed to represent the earth underneath the receiver.

If the application is mapping of a confined conductor the key features are different (Figure lb). In this case, a conductive body in a resistive host, the induction primarily occurs in and remains fixed on the conductive body $(G)$. Multiple measurements of the magnetic field, or its time-rate-of change, are made along one or more profiles (H). In this case the analysis primarily focuses on the variation of the field spatially as the geometry of the target is of most importance. The decay rate itself does yield information on the conductivity-thickness product of the body. Typica11y a final interpretation is reached by matching field observations to calculations for thin plate models (I). 


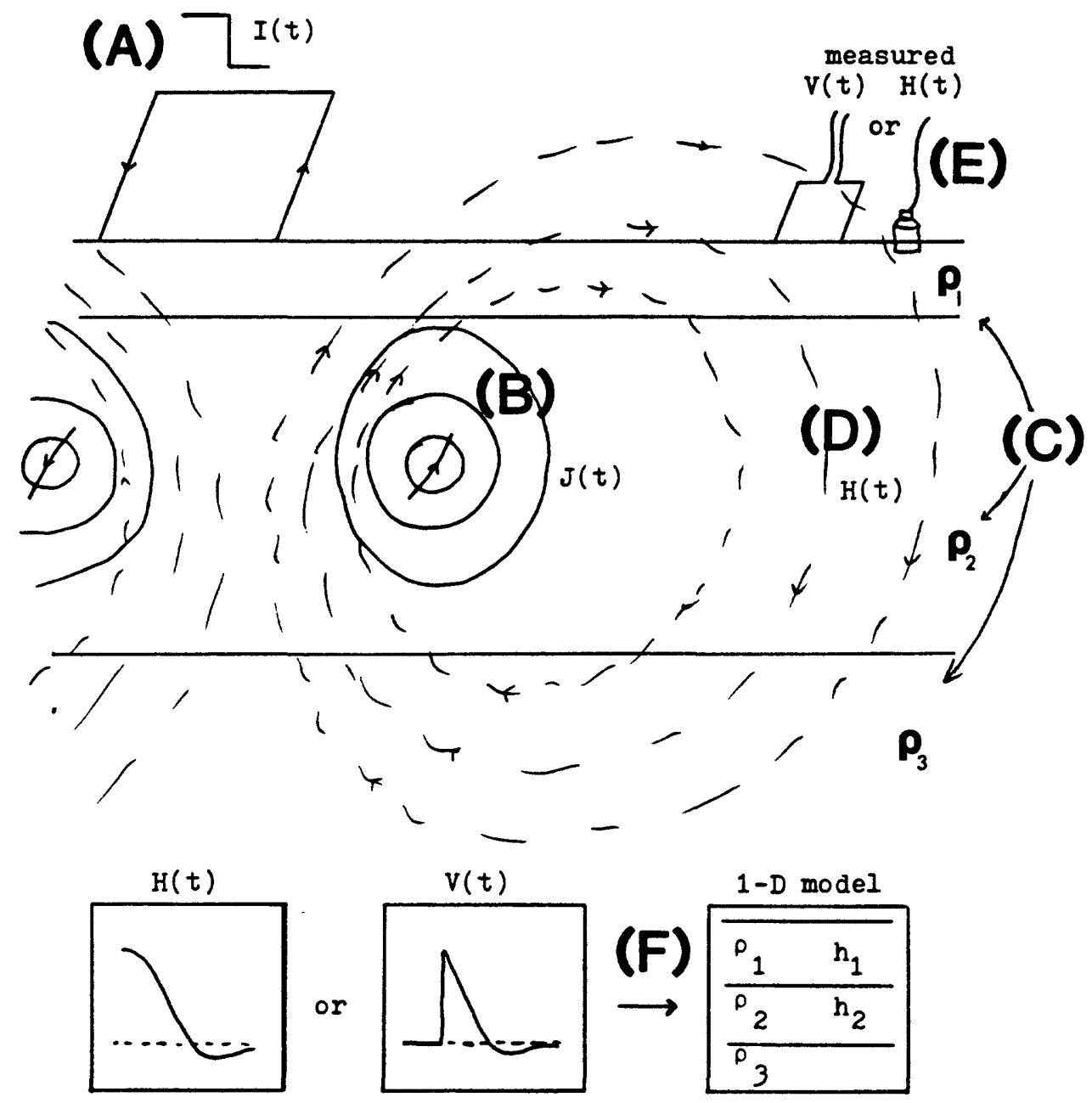

Figure 1. Cartoon synopsis of 'normal' TEM methods. Keyed features are further described in the text. (a) Features of typical TEM sounding methods: (A) truncation of current in transmitter loop, (B) currents induced in earth, illustrated with contours of current density, J, (C) the resistivity structure of the earth governs the diffusion process, (D) a time-varying secondary magnetic field is created by the diffusing currents, illustrated with dashed field lines, (E) a magnetometer measures the magnetic field or a coil measures the time-rate-of-change of the magnetic field, and (F) the data are inverted into a layered earth model. 


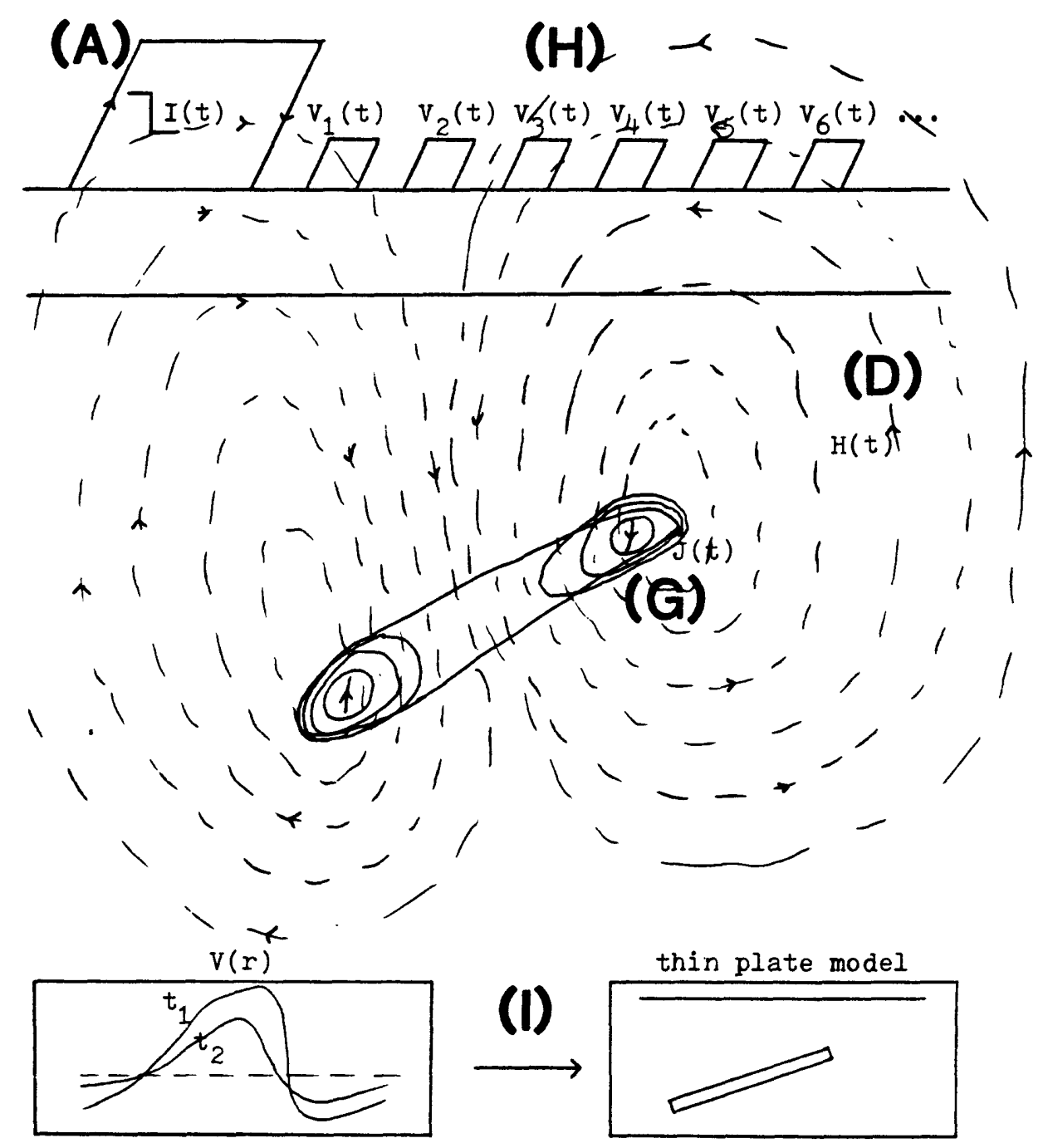

Figure 1. (b) Features of typical TEM confined conductor mapping methods: features (A) through (E), already described, are still applicable; additional features are $(G)$ the induction is primarily fixed on the conductive body, $(H)$ measurements are now made at multiple locations on profiles, and (I) the data are interpreted with thin plate models. 
Both styles of operating work well when the earth is not too different from the assumed properties. However, in the presence of more general 2-D or 3-D structures, neither process can be counted on to provide accurate results. The layered earth procedure primarily focuses on signal variation with time, with little analysis of spatial signal variations. The confined body procedure primarily focuses on spatial signal variation, with relatively less utilization of signal variations with time. More complicated structures, on the other hand, can be counted on to create significant signal variations in both space and time. At present, when an interpreter is faced with such a problem, the strong tendency is to resort to expensive, time consuming 2-D or 3-D modeling to try to approximately fit the data. Besides expense, a further pitfall of this approach is that all such programs have their limits - limits which are usually not known with certainty and not necessarily recognizable in its results. Even when used properly, the final interpretation supplied by this approach often is not geologically realistic. This state of affairs is leading many researchers into methods of imaging - processes which more directly and rapidly generate pictures of the subsurface structure from signal variations occurring in both time and space.

PART III: THE CONCEPT OF TRANSIENT ELECTROMAGNETIC IMAGING

\section{Fundamentals}

TEM measurements are usually of either the magnetic field or the voltage induced in a coil (proportional to the time rate of change of the magnetic field) as a function of time as created by a known artificial source. Normally a step current waveform is used in either a closed loop or grounded wire transmitter configuration. Since measurements are made in the time range after propagation effects have ceased, the TEM field measurement at 
any one instant in time contains contributions from currents everywhere in the earth's subsurface. However, the distribution of current density within the earth changes with time in a diffusion process. At the moment of transmitter turnoff, currents are induced in the earth that maintain the magnetic field that existed just prior to turnoff. The necessary current density distribution to accomplish this is highly concentrated very near the source in an image of the transmitter geometry. This initial current density distribution, with increasing time, diffuses into the earth and dissipates. The diffusion process is governed by the geoelectric structure of the earth. The entire history of the current density distribution is a function of the current waveform, the transmitter geometry, the vector diffusion equation,

$$
\begin{aligned}
& \nabla^{2} \vec{E}-\sigma \mu \frac{\partial \vec{E}}{\partial t}=0 \\
& \nabla^{2} \vec{H}-\sigma \mu \frac{\partial \vec{H}}{\partial t}=0
\end{aligned},
$$

and the geoelectric structure of the earth. In equation (1), $\sigma$ is the conductivity, $\mu$ is the magnetic permeability, th the electric field, and H is the magnetic field.

For the cross-sectional perspective defined in Figure 2, Plate 1 illustrates the diffusion process for an elongate loop source on a uniform halfspace of $100 \mathrm{ohm}-\mathrm{m}$ with various time slices of the current density. The currents in the earth flow in closed horizontal loops as they diffuse down and outward from the transmitter location with increasing time. In this crosssectional perspective all currents are flowing in the y-direction in either a positive or negative direction. The warm colors on Plate 1 are currents flowing out of the page and the cold colors are currents flowing into the page. The vertical lines that remain fixed, with time, underneath the transmitter location is the zero contour, or axis of symmetry. This is the 


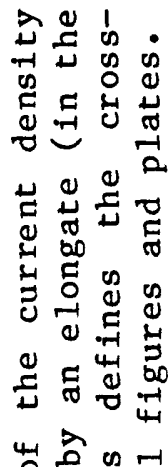

顸全步云

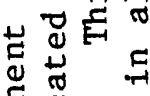

E 용

前

용

>

릉

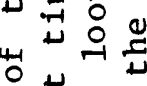

듕

艺造号

d \& $N$

P 4

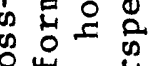

บ

동

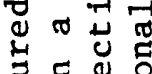

도웡

工

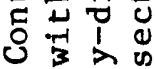

$\underset{\substack{N \\ 0}}{0}$

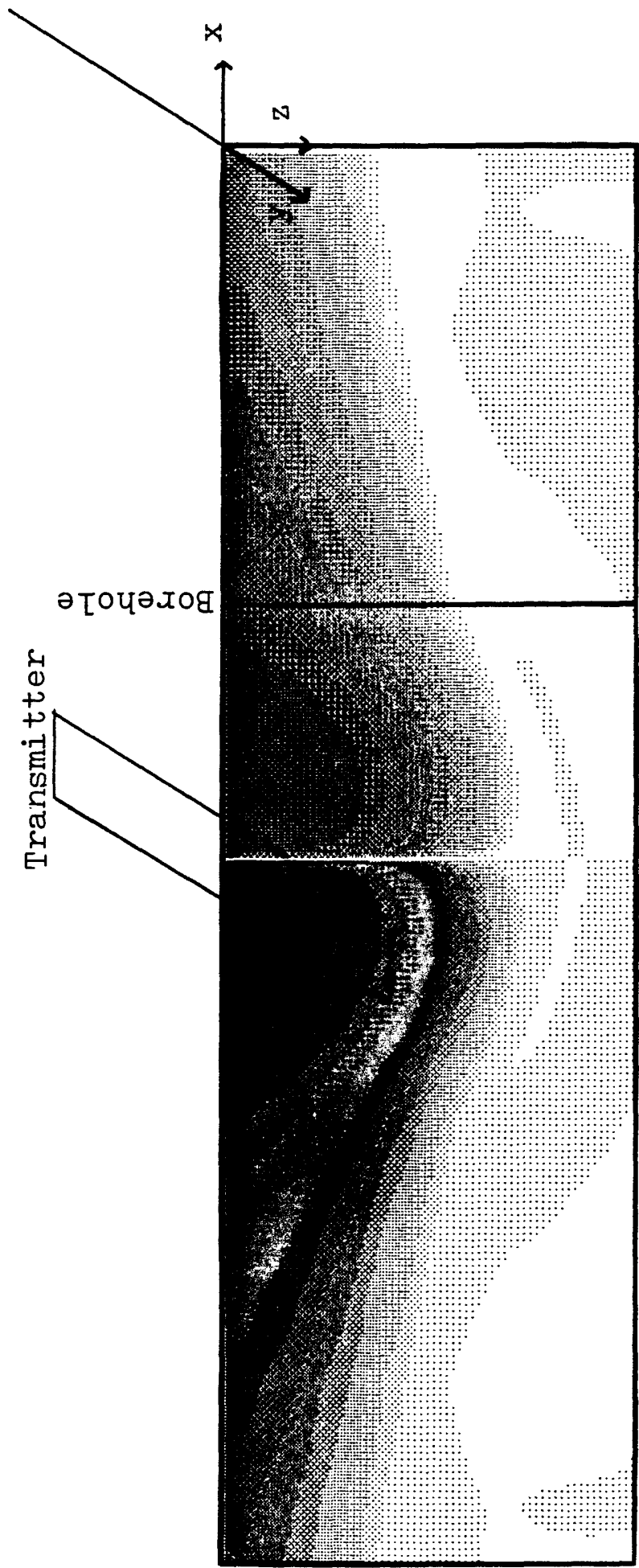


classic 'smoke ring' pattern illustrated by Nabighian (1979) and Oristaglio and Hohmann (1984).

The relationship between TEM field measurements and this current density distribution, as governed by Biot-Savart's Law for an elemental volume, is

$$
\overrightarrow{\mathrm{h}}\left(\mathrm{x}, \mathrm{y}, \mathrm{z}, \mathrm{x}^{\prime}, \mathrm{y}^{\prime}, \mathrm{z}^{\prime}\right)=\frac{1}{4 \pi} \frac{\vec{\jmath}(\mathrm{x}, \mathrm{y}, \mathrm{z}) \mathrm{x} \hat{\mathrm{r}}}{\mathrm{r}^{2}} \mathrm{dv}
$$

where $\vec{h}\left(x, y, z, x^{\prime}, y^{\prime}, z^{\prime}\right)$ is the field contribution at position $\left(x^{\prime}, y^{\prime}, z^{\prime}\right)$ from an element of current density, $\stackrel{\grave{j}}{(x, y, z)}$, at position $(x, y, z)$ at a distance $r=\left(\left(x-x^{\prime}\right)^{2}+\left(y-y^{\prime}\right)^{2}+\left(z-z^{\prime}\right)^{2}\right)^{1 / 2}, \hat{r}$ is the unit vector in the direction of $\vec{r}, d v$ is the elemental volume, and $X$ denotes the vector cross product. The measured TEM field, $\vec{H}$, is the sum of all such elemental contributions. Noting however the $1 / \mathrm{r}^{2}$ factor in Biot-Savart's Law, the effective region of significant contributions is spatially restricted. Plate 2 shows crosssections of the normalized contribution $\left(h_{z} / H_{z}\right)$ of the current densities displayed in Plate 1 to $\mathrm{H}_{z}$ at a specific location.

These cross-sections have several notable features. First, there are regions of opposing contributions to the signal. One change in sign is observed along the line underneath the transmitter position as a result of the sign change in the current density depicted in Plate 1. The other sign change occurs along the vertical line passing through the measurement position and is a result of the vector cross product in equation (2). There is zero contribution of currents to $H_{z}$ when $x=0$. The region between the transmitter and receiver contributes to $\mathrm{H}_{z}$ in opposition to the contributions of the region's exterior to the source and receiver. A sign reversal occurs in $H_{z}$ between the times of $.31 \mathrm{~ms}$ and $1 \mathrm{~ms}$ and, since $\mathrm{hz} / \mathrm{H}_{\mathrm{z}}$ is being plotted, the 
cross-sections also show this sign reversal. The pattern of signal contribution is an overprint of the current density pattern with the spatial pattern of the vector cross product and the $1 / \mathrm{r}^{2}$ geometric factor. The sustained strong contribution from elements at small $r$ illustrates the potential problem that could occur if current gathering structures occur near the measurement location. This idea for quantitative study of the area of investigation originated with Sidorov and Gubatenko (1974), Roy and Dhar (1970), Dhar (1972), and has also been applied by Kauahikaua (1982) and Patra and Ma11ick (1980, p. 117-122).

The TEM field measurements are therefore a function of transmitted current waveform, transmitter geometry, the diffusion equation, the geoelectric structure of the earth and Biot-Savart's Law. It is only the geoelectric structure of the earth that is of interest, while the other influences are factors that we wish to remove.

The main complicating influence is the diffusion process. The diffusion process, illustrated in Plate 1 , is strongly controlled by the geoelectric structure in a complicated manner. However, note two we11 known features of electromagnetic diffusion: 1) currents diffuse more slowly in conductive regions, and 2) currents dissipate less in conductive regions. Thus the assertion is made that the integral, over the duration of the transient field, of the current flowing through a subsurface volume is proportional to the element's conductivity. In all likelihood this integrated current - conductivity relationship might only be nearly linear for sma11 changes in conductivity. A second assertion is that an estimate of the total current that flows in a subsurface element can be made if data as a function of both time and position are utilized in the manner described in the following section. Finally, a representation (or image) of the geoelectric 
structure is made from the time-integrated current estimates for all elements, by normalizing by calculations of the time-integrated current, for the same elements, for a known reference geoelectric model. Figure 3 displays such a time-integrated current estimate for a uniform halfspace. If this uniform halfspace is the reference model, then this estimate ts used to normalize the estimate made from data for the 2-D structure. The large amount of spatial variation in the time-integrated current estimate apparent in Figure 3 clearly demonstrates why such a normalization is necessary. In general, the calculation of electromagnetic field quantities is easily performed only for layered earth structures. Therefore practical implementation is likely limited to use of layered structures as the reference geoelectric model. A formal proof of these assertions are not provided as it is a formidable problem and probably only provable, if at all, for special simple examples. What is more important is whether they provide a useful product in a practical way for problems of interest.

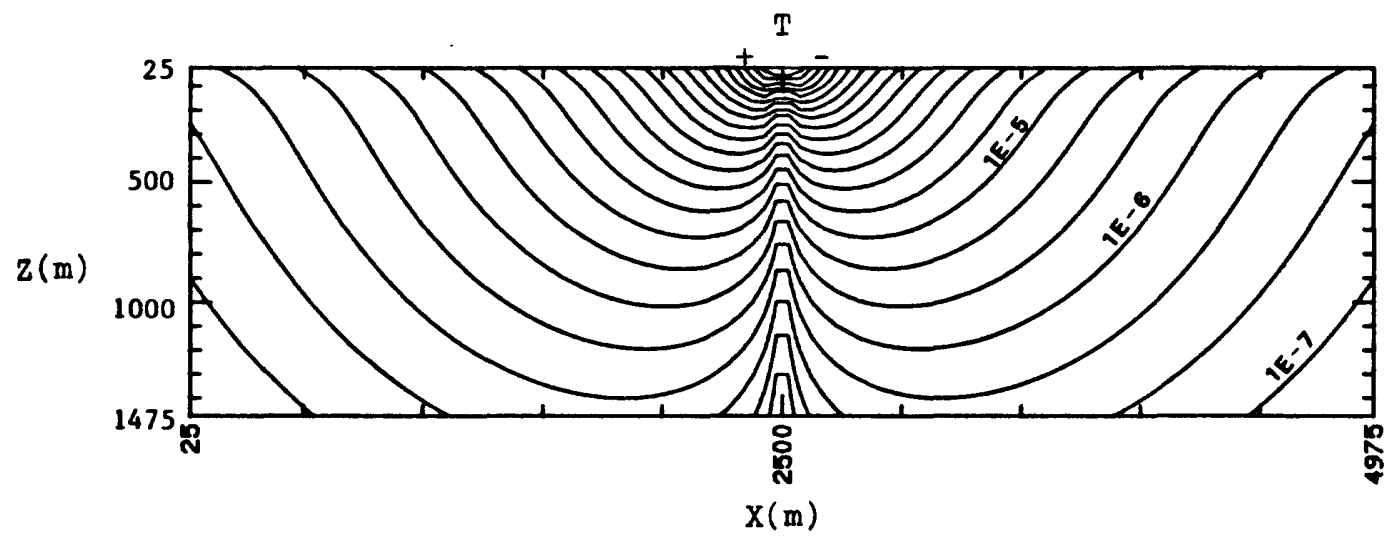

LOS CONTOUR INTERVALS

Figure 3. Cross-sectional contour plot of the sum of contributions from currents, in a 100 ohm-m uniform halfspace, to the magnetic field z-component for 41 times and 25 receiver locations in a borehole. The borehole is located at $X=2,500 \mathrm{~m}$ (coincident with center of loop source) and the receivers are at depths in the borehole from $25 \mathrm{~m}$ to $1225 \mathrm{~m}$, with a $50 \mathrm{~m}$ spacing. 
Implementation of the imaging algorithm has several we11-defined steps which are summarized in Figure 4. Primary steps illustrated in Figure 4 are keyed to the following description. The first step in implementation is to calculate the current density distribution (A) as a function of time, within the reference layered structure, for a region of the earth large enough to contain all significant contributions to the TEM field measurement locations (see, for example, Plate 1). Since at the present time the focus is on 2-D targets we will assume that an elongate pseudo-2-D source geometry is being used with the result that only a single vertical cross-section of the current density need be calculated. Next calculate a synthetic TEM field profile perpendicular to the source on the layered earth's surface and/or down a borehole (B). Now, using Biot-Savart's Law, calculate the contribution of each element of the current density distribution at each point in time to the synthetic TEM data at each measurement location. When normalized by the synthetic TEM field values these contributions become relative contributions expressable in percentages (C). Plate 2 was a previous example for a uniform halfspace and a specific borehole measurement location. Plate 3 is an example of the contribution pattern for a conductive overburden model and the same borehole measurement location. The conductive overburden is $10 \mathrm{ohm}-\mathrm{m}$ and 250 m thick over a 500 ohm-m basement. A complete relative contribution grid is four-dimensional, being a function of $x, z$, time, and receiver location.

Now assume we have a measured TEM field profile, positionally coincident with the synthetic profile, for an earth containing some twodimensional geoelectric structure (D). Here we use the relative contribution grids as a weighting coefficients to extract fractions of the measured TEM 


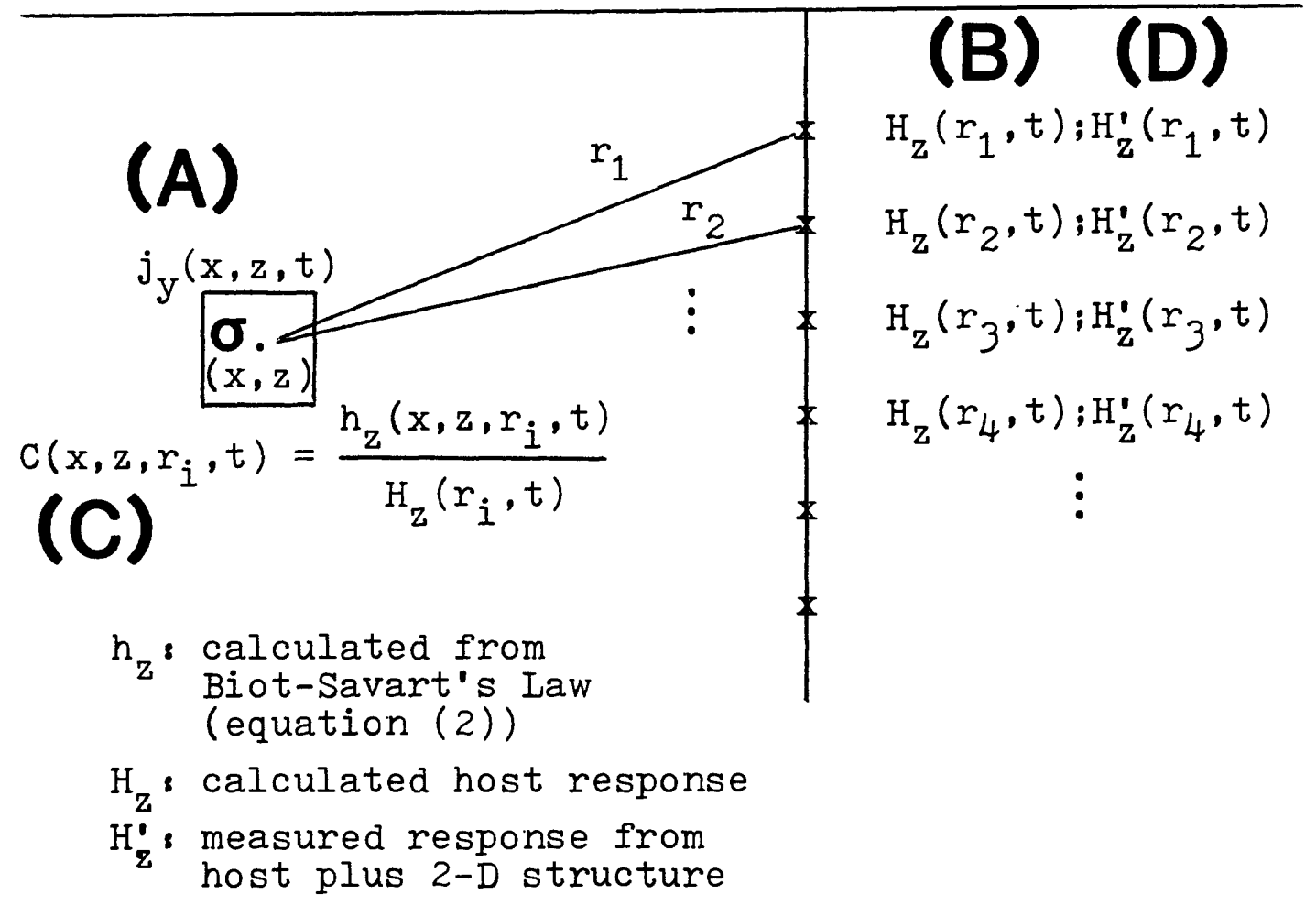

$$
\begin{aligned}
& \text { (E) } 2 D(x, z)=\sum_{r_{i}} \sum_{t} C\left(x, z, r_{i}, t\right) \cdot H_{z}^{\prime}\left(r_{i}, t\right) \\
& \text { (F) } 1 D(x, z)=\sum_{r_{i}} \sum_{t} C\left(x, z, r_{i}, t\right) \cdot H_{z}\left(r_{i}, t\right) \\
& \text { (G) } \sigma_{a}(x, z)=\frac{2 D(x, z) \cdot \sigma(x, z)}{1 D(x, z)}
\end{aligned}
$$

Figure 4. Synopsis of the TEM imaging algorithm. Keyed features are further described in the text. Primary steps are: (A) calculation of current density for reference layered earth model, (B) calculation of magnetic field on profile for that reference model, (C) calculation of relative contribution of current to the magnetic field for the reference model, (D) measured magnetic field on profile for earth containing 2-D structure, (E) calculate timeintegrated current estimate for earth containing 2-D structure, (F) calculate time-integrated current estimate for reference model, and (G) calculate estimate of conductivity for 2-D earth by ratioing the two time-integrated current estimates and multiplying by the reference conductivity. 
field values at all points in time and all receiver positions to be assigned to each subsurface element. The fractions for each subsurface element are summed to effect a stacking procedure in both time and space (receiver location) (E). This stacking is also performed with the synthetic data to establish the pattern for the reference model (F). This reference stack is used to normalize the stacked values for the measured data. Figure 3 presented an example of a reference stack for the case of a borehole magnetic field profile in a uniform earth. This normalization provides a crosssectional image, by no means unique, however, of where the strong and weak contributions are located with respect to the contribution pattern of the reference mode1. Multiplication of this contribution image by the reference conductivity structure (G) then creates a cross-sectional image of the earth's apparent conductivity which can be graphically displayed. This procedure can be further generalized to incorporate multiple source locations.

One important aspect of the present imaging procedure is the need to mask out a narrow time window from the summing process. This is due to the sign change that occurs in the magnetic field for any geometry in which the source and receiver are laterally displaced from one another. This sign change marks the time at which the "equivalent current filament" - the single closed filament of current which can create the same magnetic field at the measurement point (Nabighian, 1979) - passes from one side of the receiver to the other. In other words the majority of contribution to the signal now comes from the farther side of the receiver. This zero in the magnetic field creates a singularity in the weighting coefficients because their calculation involves a normalization by the magnetic field. Weighting coefficients near this singularity are a couple of orders of magnitude larger than at other times. Inclusion of these abnormally large weighting coefficients 
creates large imaged resistivity anomalies from small anomalies in the data large enough to dominate the real anomaly. Therefore this mask is always needed as the algorithm now stands.

As demonstrated by testing summarized in the following section, the quality of result achievable with this TEM imaging algorithm is strongly dependent on how accurately the selected reference model is chosen. It is necessary to have a good estimate of the background layered structure to achieve good resolution of the two-dimensional structure. It may be possible to create a mechanism within the imaging procedure to automatically generate a good estimate of the background layered structure, but at present this has not been accomplished. Therefore, the reference model must be externally supplied.

PART IV: TESTING OF THE TEM IMAGING ALGORITHM FOR TUNNEL DETECTION Tunnel in Uniform Halfspace - Loop Source

The results presented here are for elongate loop sources and $z^{-}$ component magnetic field measurements in boreholes. The modeled tunnel is 120 meters long and the source loops are 48 meters along strike and 24 meters wide. The boreholes lie on the cross-sectional plane through the center of both the rectangular source loop and the tunnel so, by symmetry, no galvanic effects are present. All calculations were performed using the 3-D modeling program by Newman et al. (1986).

Effect of source-borehole separation and survey design

Initial modeling results indicated that, for a single tunnel at a particular depth, there is little to be gained by use of multiple sources and only a single borehole for the purpose of creating a subsurface image. There is a dependence on the distance between the source and borehole for the 
amplitude of the anomaly but this does not benefit imaging of the structure. However, there is a single optimum source-borehole separation that depends on the position of the target. The ideal situation is to locate the source such that the current density maximum passes through or very near the anomalous zone, thus maximizing the anomaly in the EM field. Referring again to Plate 1 , it can be seen that the depth range that lies in the path of current density maximum becomes deeper as transmitter-borehole separation increases. Given that the position of the tunnel is unknown to begin with it becomes necessary to approach the problem with a profile of multiple sources and boreholes. Each transmitter-borehole offset utilized will illuminate a different depth range in the borehole (Figure 5a). Ideally a regularly spaced profile of boreholes is needed to construct an image and source spacings should coincide with borehole spacings. The recommended survey design is presented in Figure $5 \mathrm{~b}$.

Processing of each transmitter-borehole profile data set individually through the imaging algorithm indicates the optimum sourceborehole spacing to utilize for the image construction for a target at a particular depth. A tunnel with conductive shell model is shown in Figure 6. The variation of anomaly amplitude with transmitter-borehole separation is shown in Figure 7 for this model. The loop centers are 48, 72, and 96 meters away from the borehole. The images are constructed using weighting coefficients calculated for the uniform halfspace of $100 \mathrm{ohm}-\mathrm{m}$. The maximum anomaly occcurs for the source-borehole spacing of 72 meters. The final image is then constructed using all data sets along the profile which use this spacing. 


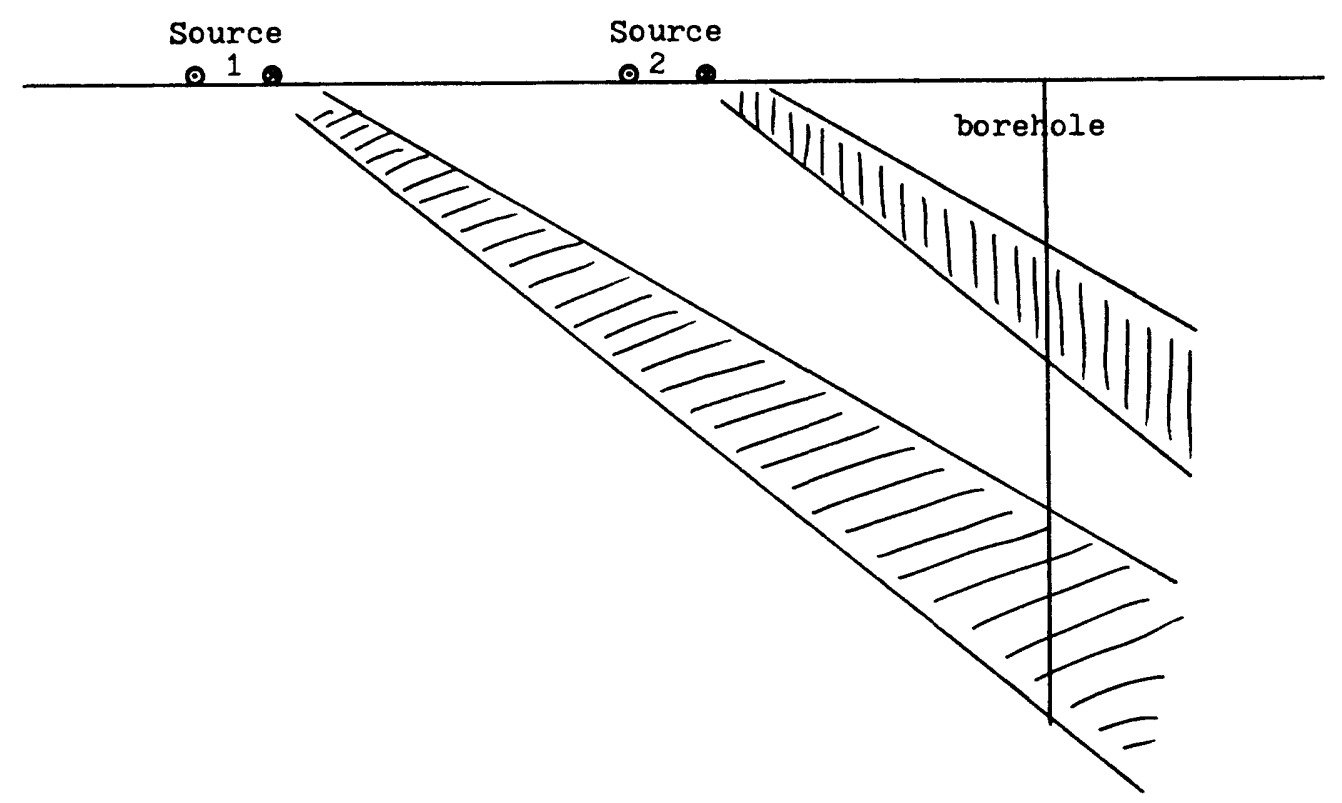

Figure 5. (a) Sketch of path of current density high, in a uniform earth, for sources at different locations. Each path is the zone of optimum target illumination for that source. This indicates that, in order to optimally illuminate the entire depth range of a borehole, multiple sources are needed.

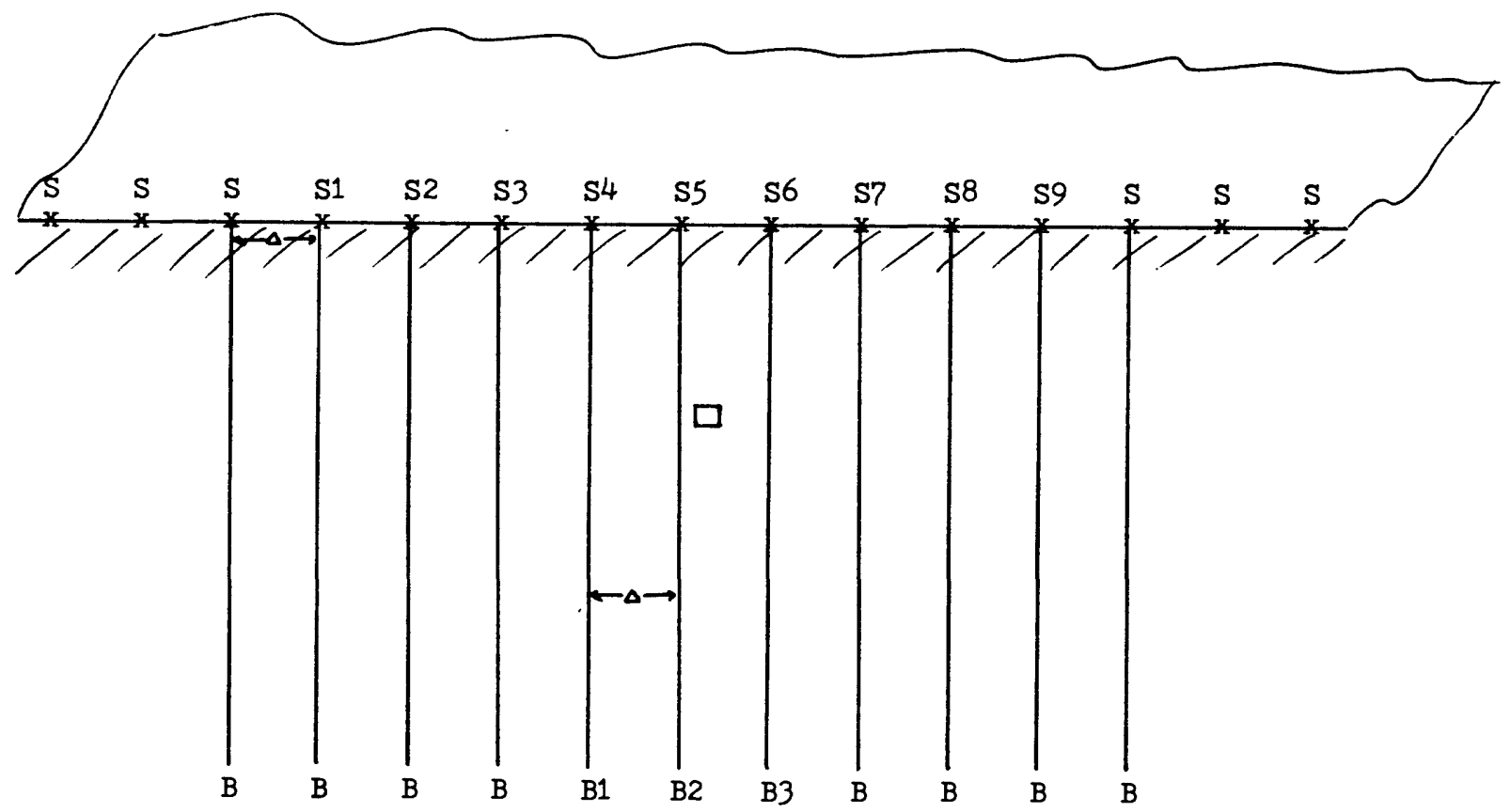

(b) The resulting optimal survey design for subsurface TEM imaging with surface sources and borehole receivers. The sources and boreholes referred to in the model study to follow are numbered. The spacing used in the model study is $24 \mathrm{~m}$ between boreholes and sources. 
Tunnel with conductive shell model

A series of six images along a profile for the conductive shell model of Figure 6 is presented in Figure 8. Each borehole is associated with two images, one for a source on either side of the borehole. First, notice that the anomaly maximum is always centered on the borehole. This is due to the nature of the weight coefficients and their strong dependence on the $1 / \mathrm{r}^{2}$ factor in Biot-Savart's Law. Also of importance is the opposite anomaly sense between the results for any one borehole for the two sources. The pattern is that the conductive shell creates a conductive anomaly when the target lies outside of the source-borehole configuration $\left(\mathrm{H}_{z}\right.$ increases), an apparent resistive anomaly when the target lies within the source-borehole configuration ( $\mathrm{H}_{\mathrm{z}}$ decreases). This behavior is due to the opposing

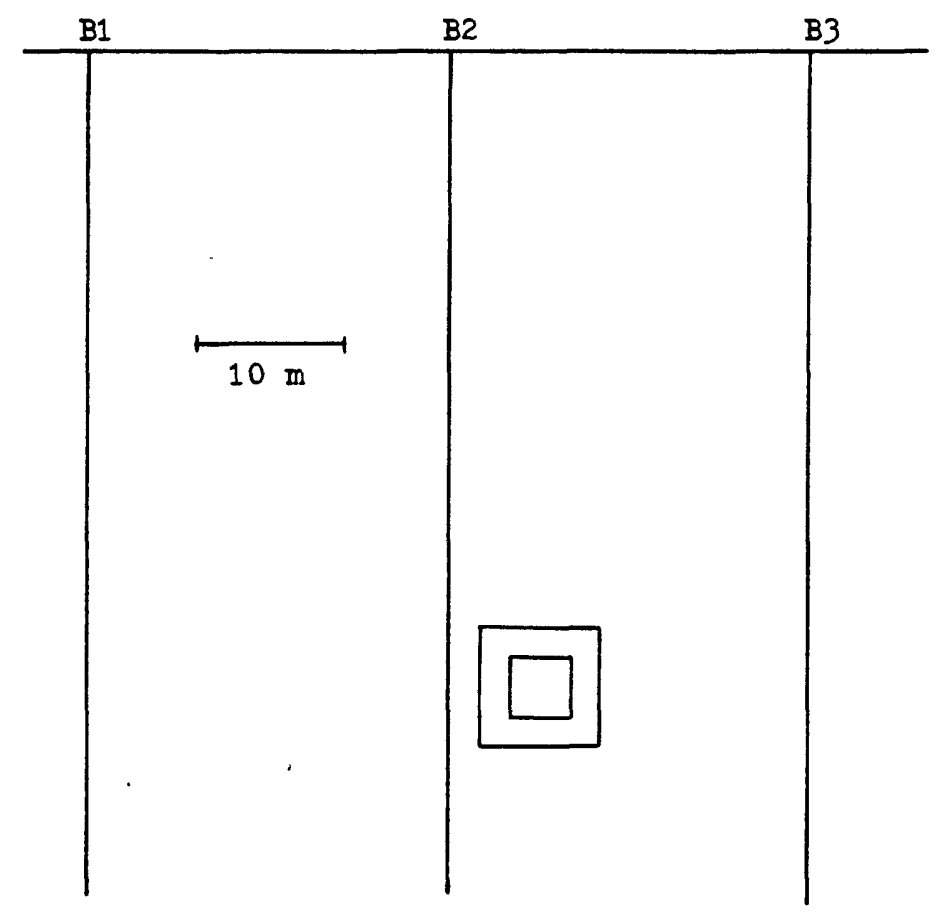

Figure 6. A tunnel with conductive shell model. The boreholes are $24 \mathrm{~m}$ apart, the tunnel is $4 \mathrm{~m}$ across, and the shell is $8 \mathrm{~m}$ across and 2 $\mathrm{m}$ thick. Borehole $\mathrm{B} 2$ is $2 \mathrm{~m}$ away from the she1l. The host rock is $100 \mathrm{ohm}-\mathrm{m}$ and the she11 is $30 \mathrm{ohm}-\mathrm{m}$. The depth to the top of the she11 is $38 \mathrm{~m}$. 


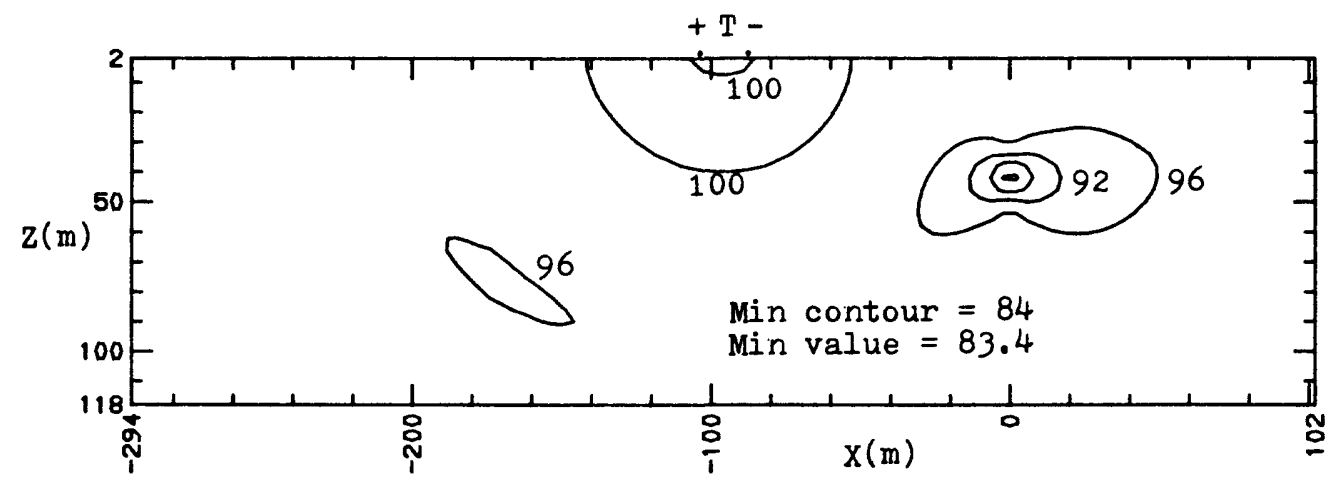

(a)

TUNAEL 3 3, SRC 1, BHOLE 2 CONTOUR INTERVAL $=4$ OHWM

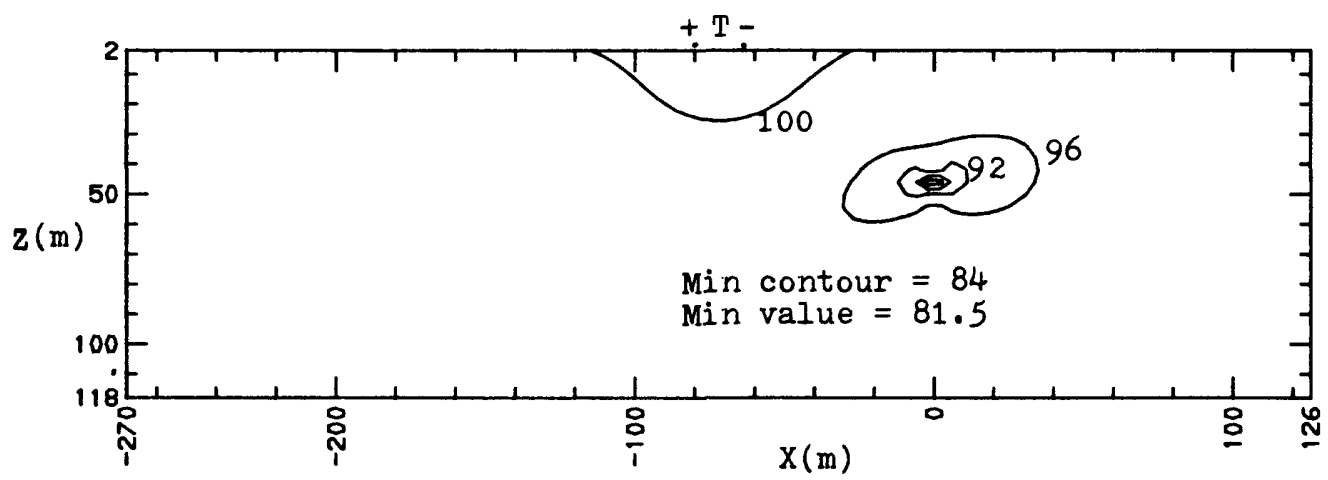

(b)

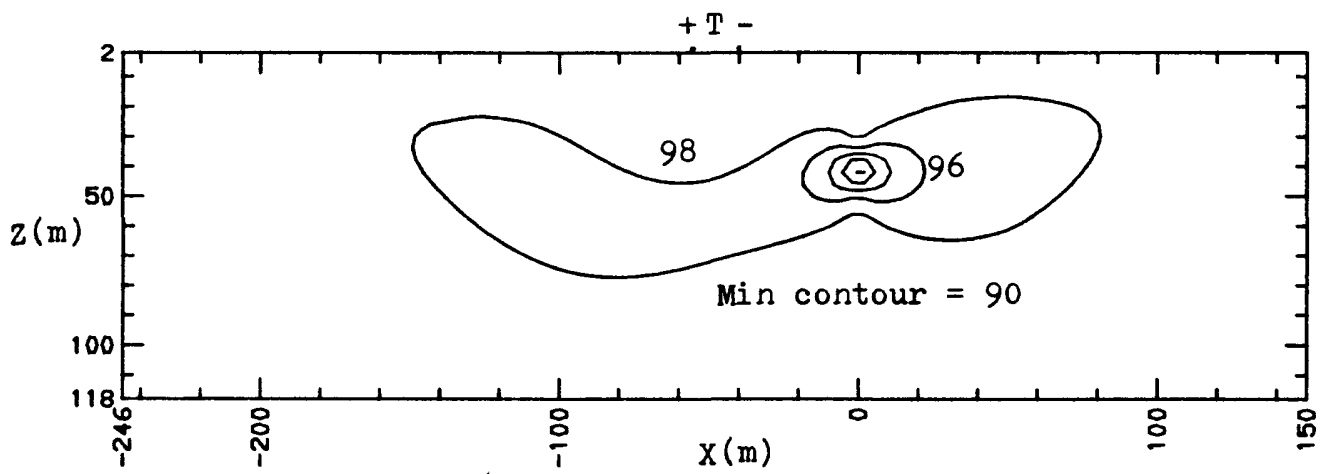

(c)

TUNNEL 33 , SRC 3, BHOLE 2 CONTOUR INTERVAL $=2$ OHM-M

Figure 7. Imaged apparent resistivity cross-section for the model of Figure 6 and three different source center - borehole separations. Tr ansmitter loop position is marked by + and -. Borehole B2 is at position $X=0$. Images for (a) source $\mathrm{S} 1$ and borehole B2 with separation $=96 \mathrm{~m}$, (b) source S2 and borehole B2 with separation $=72 \mathrm{~m}$, and (c) source $\mathrm{s} 3$ and borehole B2 with separation $=48 \mathrm{~m}$. 


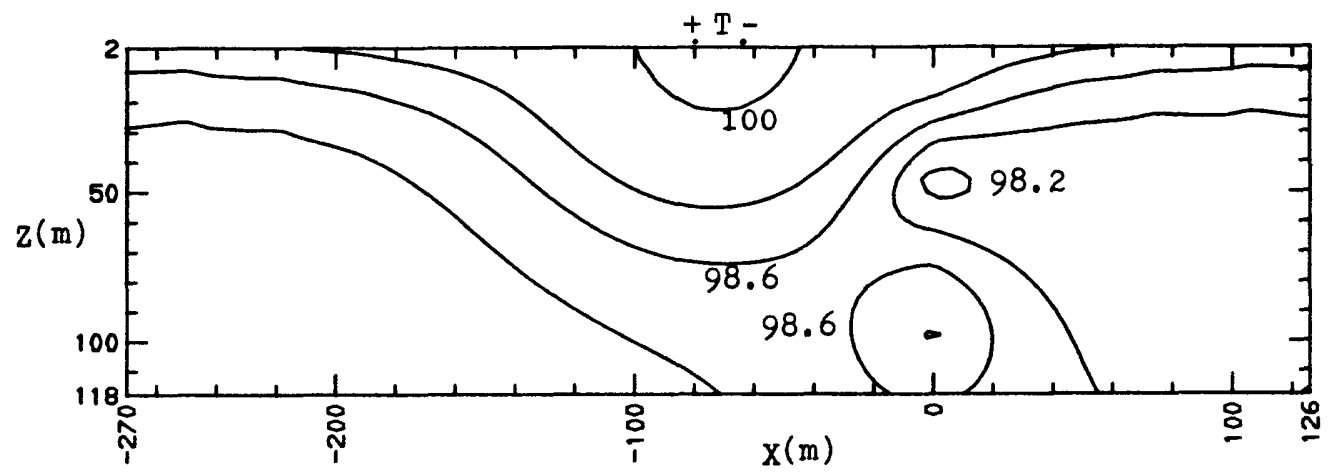

(a)

TUNNEL *3, SRC 1, BHOLE 1 CONTOUR INTERVAL $=.2$ OHM-M

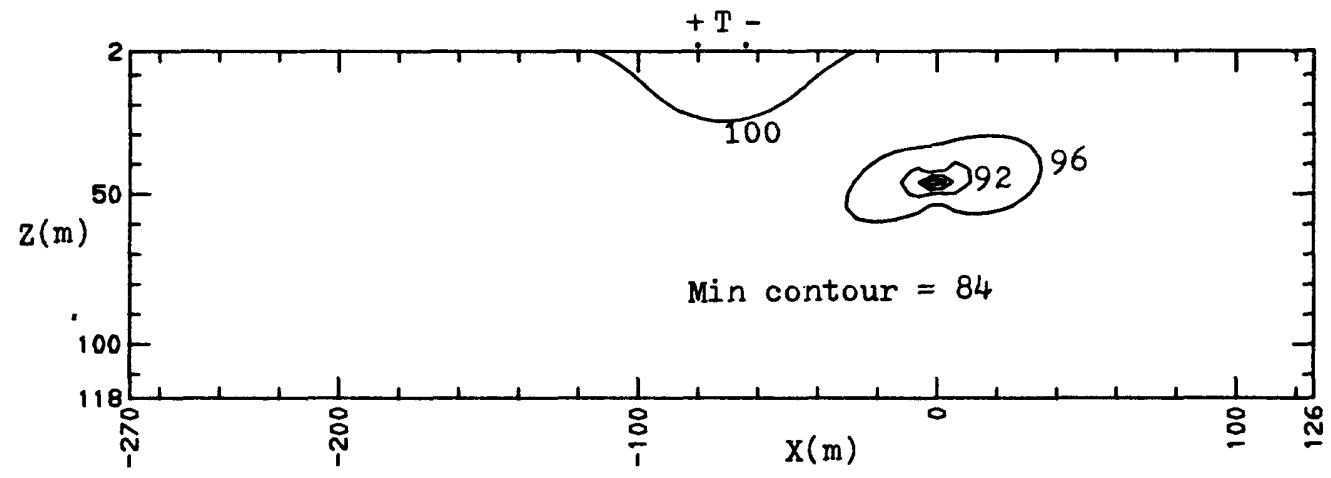

(b)

TUNNEL \#3, SRC 2, BHOLE 2 CONTOUR INTERVAL $=4$ OHM-M

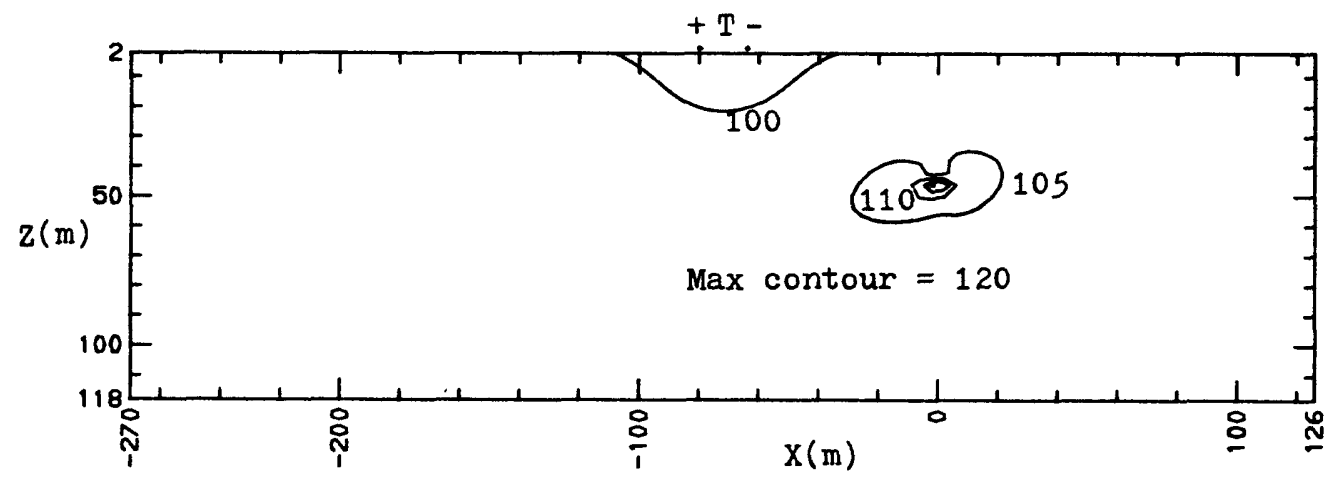

(c)

TUNNEL \#3, SRC 3, BHOLE 3 CONTOUR INTERVAL $=5$ OHM-M

Figure 8. Imaged apparent resistivity cross-section for the model of Figure 6 for six different source - borehole combinations, all with a separation of $72 \mathrm{~m}$. The borehole is always placed at $X=0 \mathrm{~m}$. Images for (a) source $\mathrm{S} 1$ and borehole $\mathrm{B1}$, (b) source $\mathrm{S} 2$ and borehole B2, and (c) source S3 and borehole B3. 


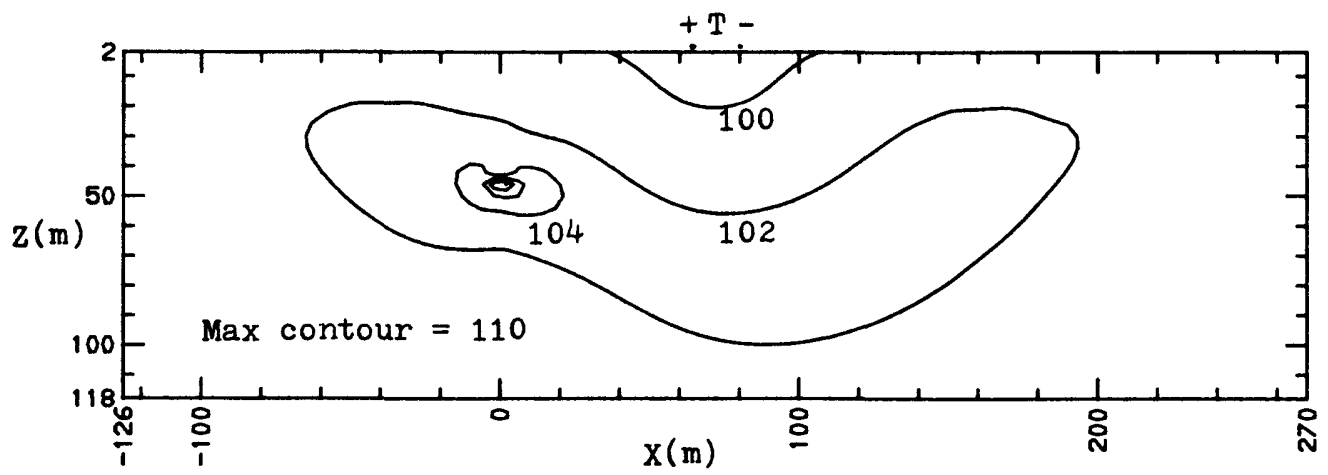

(d)

TUNNEL 33, SRC 7, BHOLE 1 CONTOUR INTERVAL $=2$ OHM-M

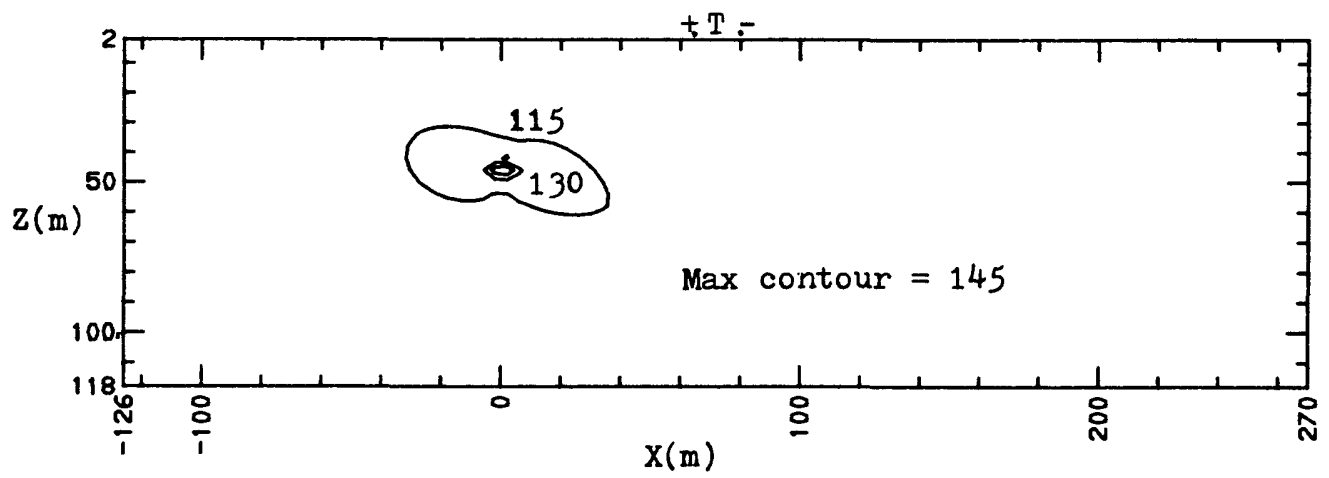

(e)

TUNNEL *3, SRC 8, BHOLE 2 CONTOUR INTERVAL = 15 OMM-M

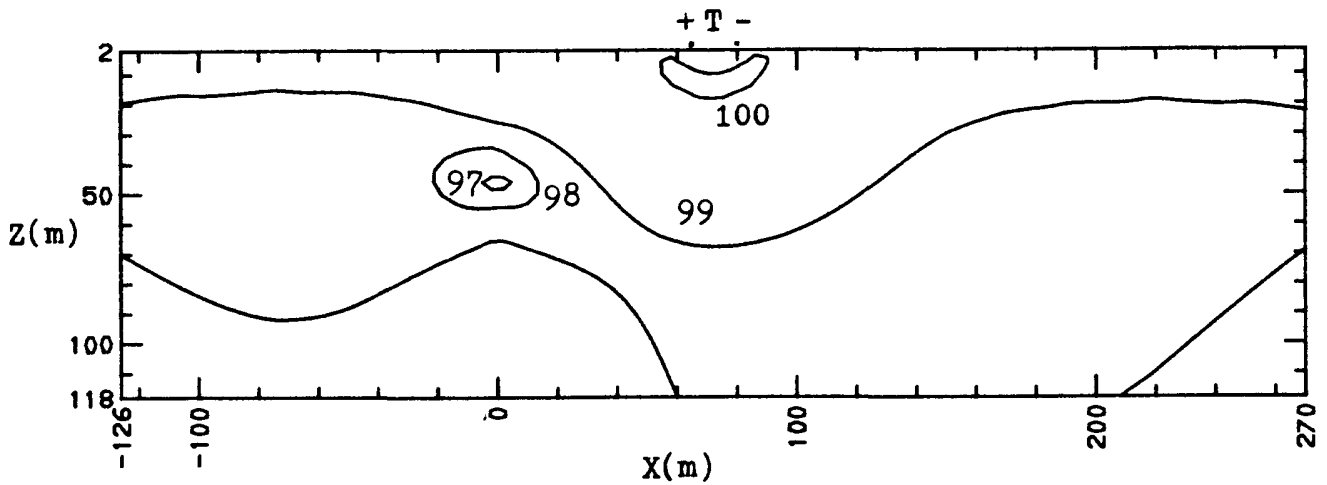

(f)

TUNNEL 3 . SRC 9. BHOLE 3 CONTOUR INTERVAL $=1$ OHMTM

Figure 8. (continued) Images for (d) source 57 and borehole B1, (e) source S8 and borehole B2, and (f) source S9 and borehole B3. 
contributions of currents in those zones to the measured field as dictated by the cur1 operator in Biot-Savart's Law. These patterns are displayed in Plates 2 and 3. This pattern will exist for any source-receiver configuration (surface or borehole) in which there is lateral separation. The pattern will disappear if the source and receiver lie on the same vertical axis. However this geometry is not attractive for tunnel detection as the normal current diffusion pattern couples only very weakly with a target nearly directly underneath the transmitter; i.e., near the current density null. There is very little anomalous response created with this geometry. So lateral separation between the source and borehole is necessary and the anomaly reversal phenomenon it creates must be accommodated.

When looking at the entire profile of results it seems likely that it is possible, in practice, to distinguish the anomalous zone (between which two boreholes the anomaly is located) based on the pattern of anomaly reversal in the rofile of borehole data sets. In constructing a final composite image from this profile there are two options. One option would be to simply exclude the images displaying the false resistive anomalies. The second option, and the one actually taken, is to estimate the conductive anomaly for those images displaying the false resistive anomaly. The method used to estimate the conductive anomaly, for each element in the grid, is to equate $\rho_{\mathrm{c}} / \rho_{\mathrm{b}}=\rho_{\mathrm{b}} / \rho_{\mathrm{r}}$, where $\rho_{\mathrm{b}}$ is the background reference resistivity of the element (from the assumed host structure), $\rho_{r}$ is the apparent resistivity of the element for the resistive anomaly, and $\rho_{c}$ is the desired apparent resistivity of the element for the conductive anomaly estimate. Since $\rho_{b}$ and $\rho_{\mathrm{r}}$ are known, we have $\rho_{\mathrm{c}}=\rho_{\mathrm{b}}^{2} / \rho_{\mathrm{r}}$. Figure 9 displays the result of this conductive anomaly estimation procedure for the original false resistive anomaly displayed in Figure $8(e)$. 


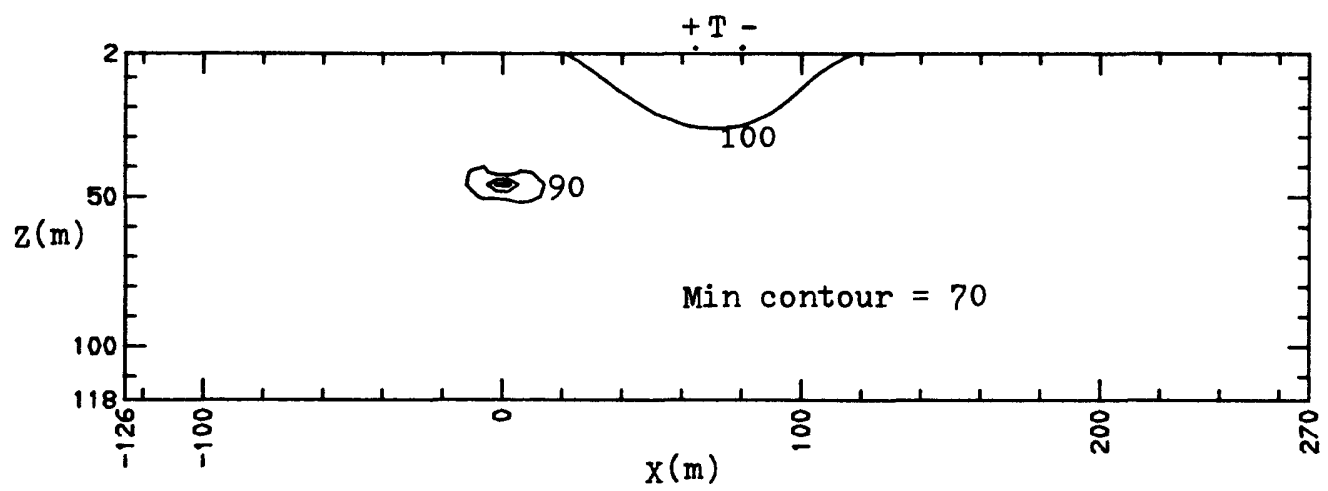

(a) TUNNEL $\$ 3$, SRC 8 , BHOLE 2

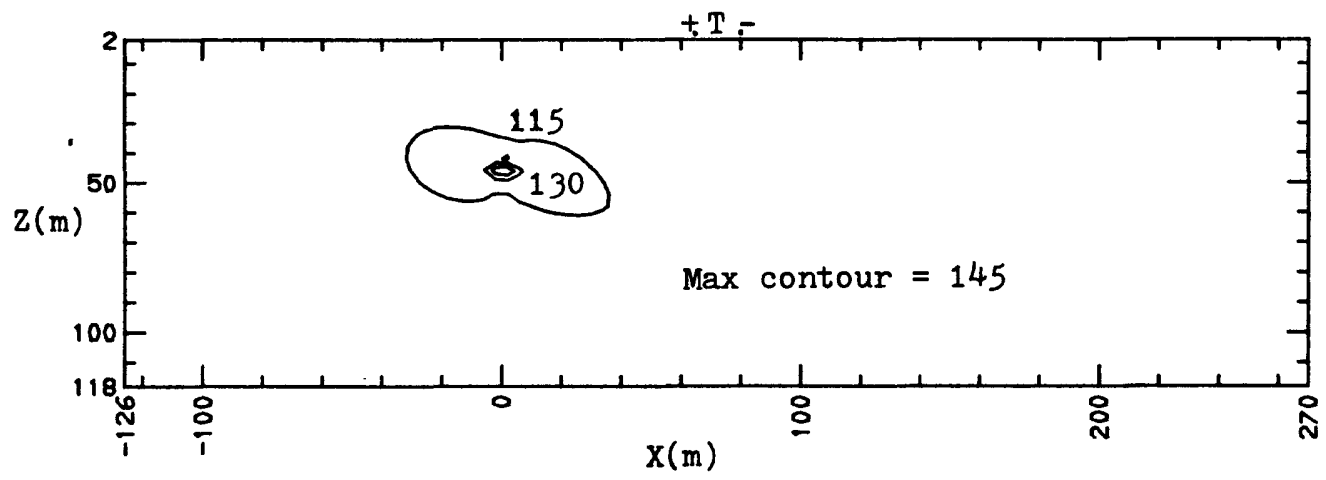

(b)

TUNNEL 3. SRC 8, BHOLE 2 CONTOUR INTERVAL $=15$ OHM-M

Figure 9. (a) Imaged apparent resistivity cross-section for source $\mathrm{S} 8$ and borehole B2 resulting from the conductive anomaly estimation procedure described in the text. (b) The original image displaying a false resistive anomaly. 
Now all six images on the profile can be reprocessed into a single composite image. The composite image is created by repeating the stacking process for all six data sets en masse, including the conductive anomaly estimation process for data sets (c), (d), and (e). This result is presented In Figure 10 for the conductive she1l tunnel model of Figure 6. Image asymmetry correctly indicates that the target is actually displaced slightly to the right of borehole B2. Figure 11 displays the geometry for the tunnel shifted farther away from borehole B2 such that the distance from borehole B2 to the edge of the conductive she11 is now 6 meters. The composite image for this case is shown in Figure 12. Again the asymmetry indicates the target is closer to borehole 2 but not by as much as the previous case. If the target were exactly in between two boreholes the anomaly would be symmetric about that location. An obvious refinement would be an algorithm to put the anomaly more nearly' in its correct lateral position as opposed to the present situation where the anomaly peaks are at the borehole locations.

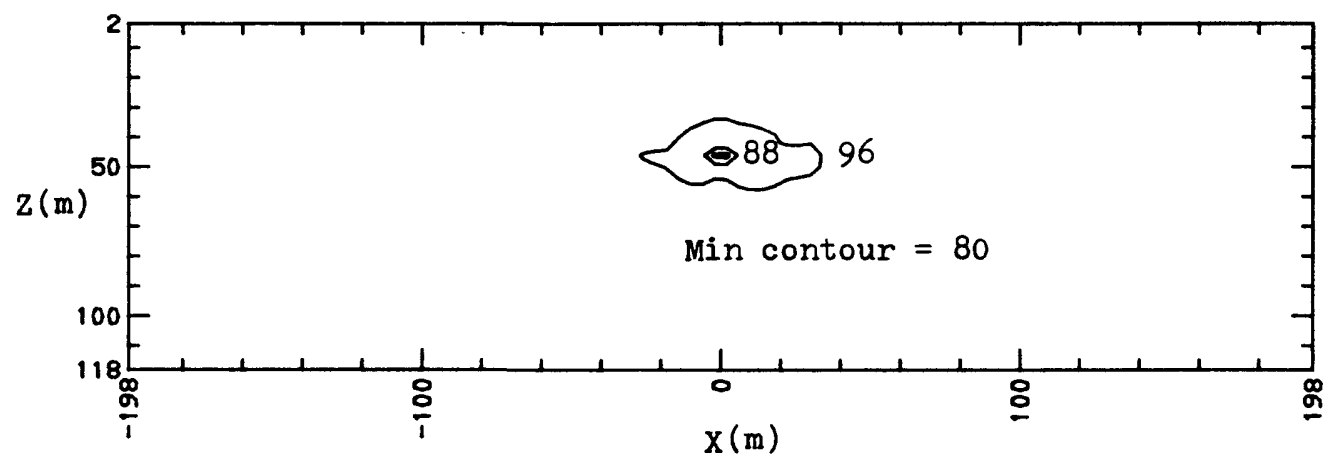

TUNNEL 33 COMPOSITE IMAGE CONTOUR INTERVAL $=8$ OHM-M

Figure 10. Composite apparent resistivity image incorporating all six data sets presented in Figure 8 . Borehole B2 lies at position $X=0$ $\mathrm{m}$. The true lateral position of the center of the target is $X=6$ m. 


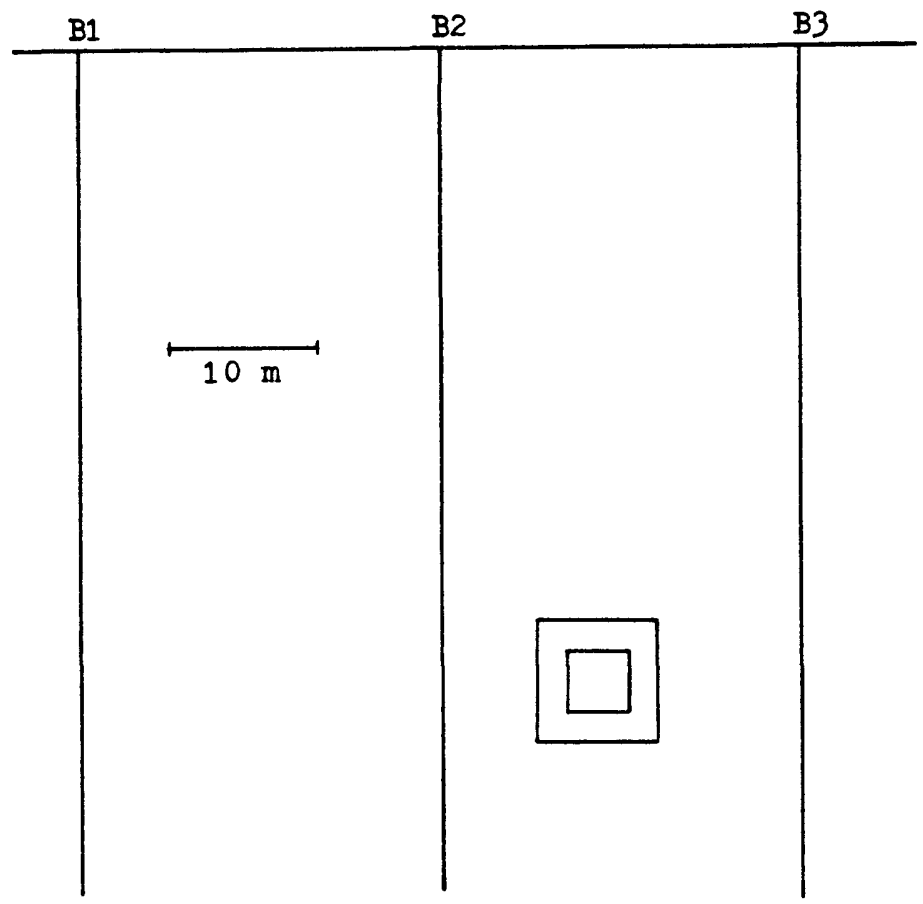

Figure 11. A tunnel with conductive she11 model identical to Figure 6 except the distance from borehole B2 to the she11 is now $6 \mathrm{~m}$.

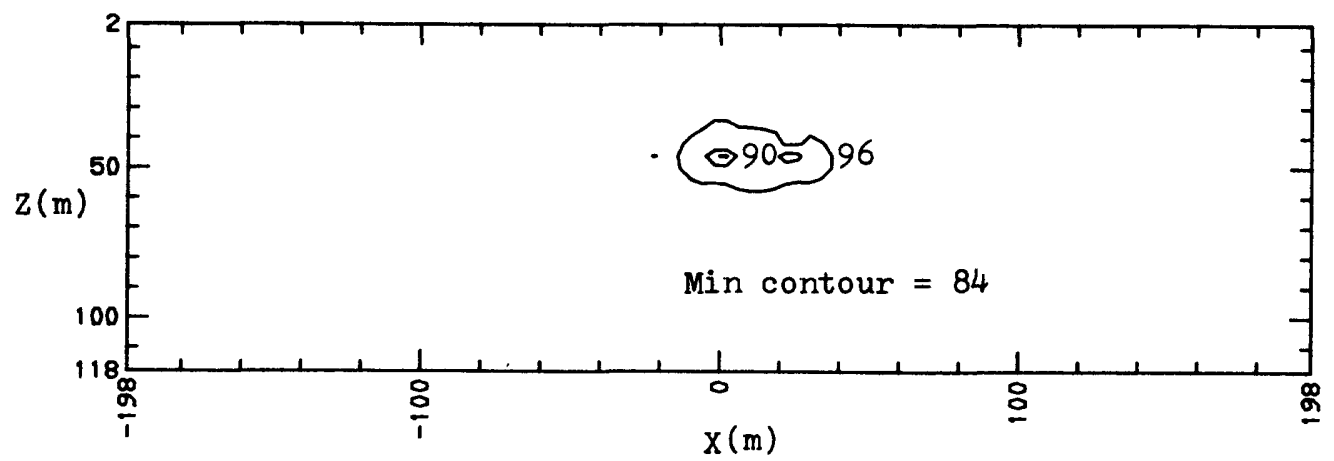

TUNNEL $* 5$ COMPOSITE IMAGE CONTOUR INTERVAL $=6$ OHWH

Figure 12. Composite apparent resistivity image for model of Figure 11. The true lateral position of the target center is now at $X=10 \mathrm{~m}$. 
The present imaging scheme produces an image anomaly (in resistivity) that is about one third the maximum anomaly seen for any one receiver in the borehole closest to the target. The scheme therefore can somehow be better tuned for anomaly enhancement. In any event it looks promising that tunnels with conductive shells can be detected in reasonably uniform host rocks. At this point the limiting influence of geologic noise is unkn own

Tunnel with no conductive shel1 model

The case of a tunnel with no conductive shell (Figure 13) is not so promising. The composite image for this model is displayed in Figure 14. The anomaly is approximately $5 \%$ and this is probably approaching the limit of practical detectibility even in advantageous geologic environments. The maximum single receiver anomaly is approximately $15 \%$. The imaged anomaly decreases to less than $3 \%$ as the tunnel is moved toward the middle of the two boreholes. A test was also made for the case of a strike direction offset between source and borehole profiles with the idea of creating galvanic effects that would enhance the anomaly. However, no improvement was discerned.

\section{Tunnel in Un iform Halfspace-Grounded Wire Source}

The result for a grounded wire source with the present imaging algorithm is not encouraging. For the model displayed in Figure 15 (same model as Figure 11) the result is the image displayed in Figure 16. The anomaly is very much less than for the loop source case (Figure 12). With a grounded wire source the smaller separation from the borehole is used because the anomaly weakens with increasing separation. It is not likely that the grounded wire source is fundamentally this much poorer than the loop source. What is true is that the two types of sources create distinctly different 


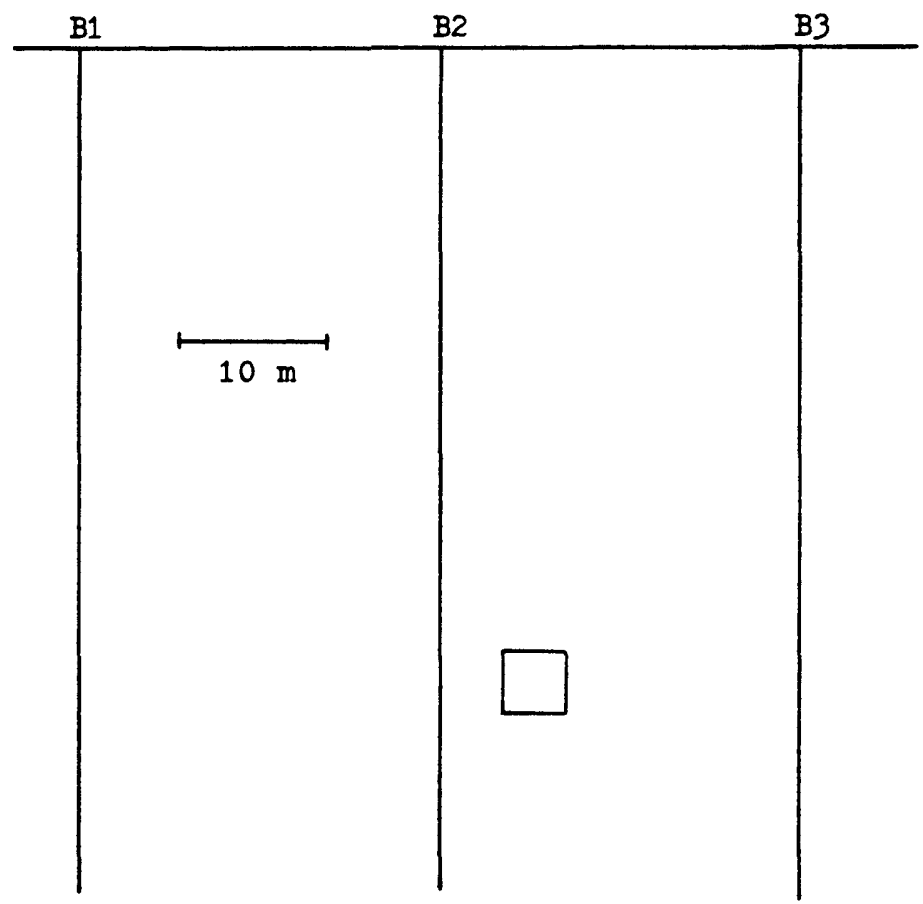

Figure 13. Model of a tunnel without a conductive shell. The tunnel is $4 \mathrm{~m}$ across and $4 \mathrm{~m}$ away from borehole $\mathrm{B} 2$. The host rock is again 100 ohm-m. The depth to the top of the tunnel is $40 \mathrm{~m}$.

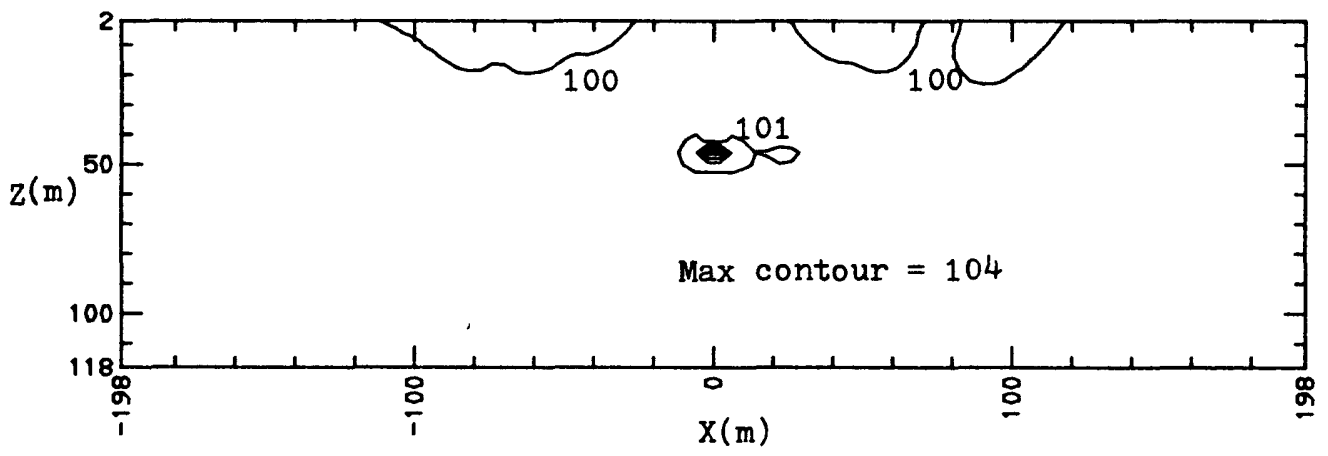

TUNNEL 44 COMPOSITE IMAGE CONTOUR INTERVAL $=1$ OHM-M

Figure 14. Composite apparent resistivity image for model of Figure 13. The true lateral position of the tunnel center is at $X=6 \mathrm{~m}$. 


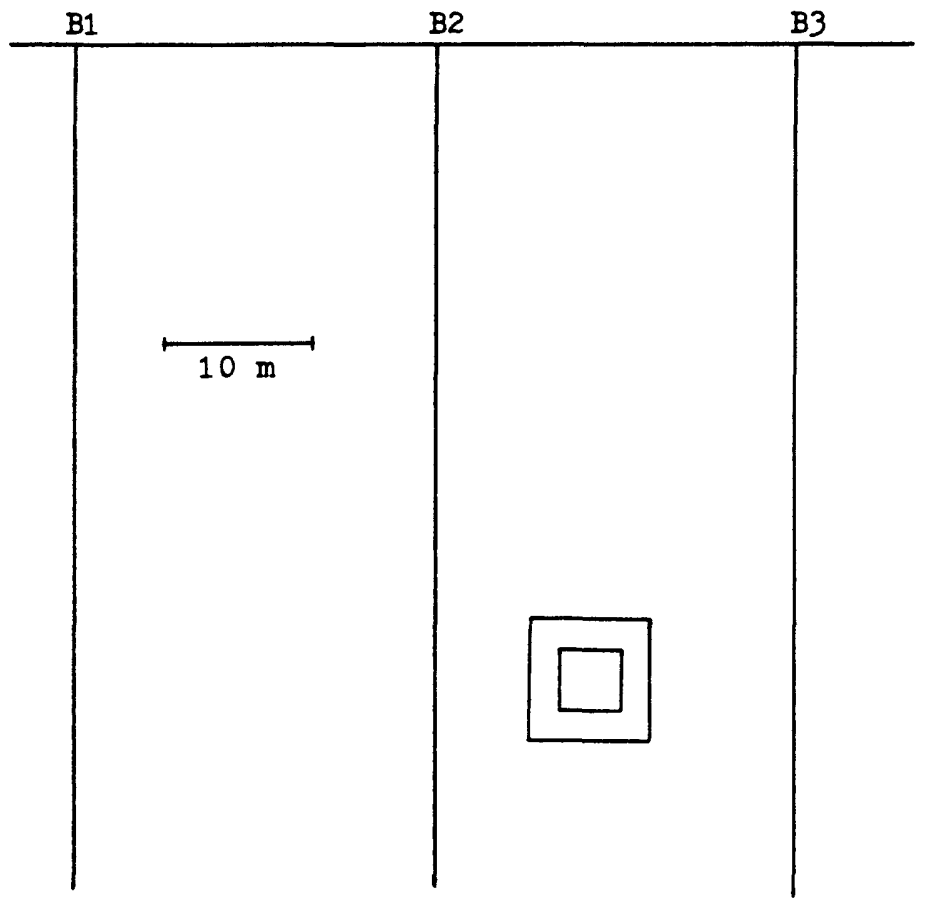

Figure 15. A tunnel with conductive shell model, repeated from Figure 11, for imaging from a grounded-wire source located at the top of borehole Bl running parallel to the tunnel.

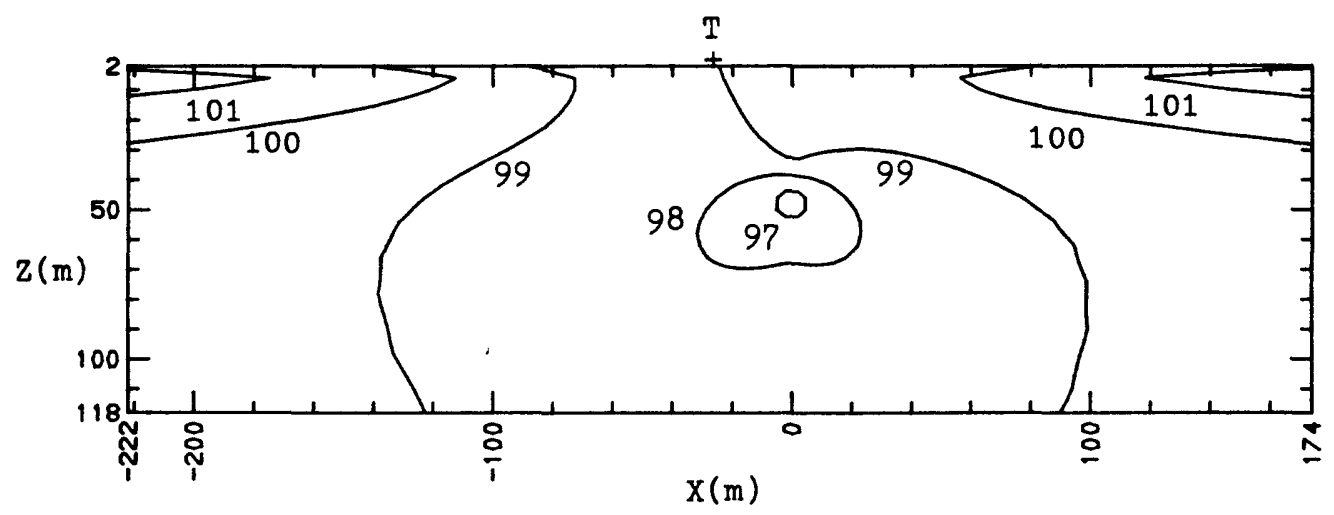

TUNNEL H5, SRC 4, BHOLE 2

CONTOUR INTERVAL $=1$ OHM-M

Figure 16. Imaged apparent resistivity cross-section for the model of Figure 15, grounded-wire source $\mathrm{S} 4$ and borehole B2. Transmitter position is marked by the + . 
patterns of current flow in the subsurface. It is likely that the present imaging algorithm is in some way tuned for the loop source case. Research on an effective variation for the grounded wire source is obviously needed.

\section{Tunne1 beneath Conductive Overburden-Loop Source}

With a layered host rock there is a choice available for the imaging algorithm to use weighting coefficients calculated for either the layered model or a uniform halfspace with resistivity equal to the first layer resistivity. The ideal case where the layered structure is known and is used for the weighting coefficient calculations, and using the layered earth magnetic field data, yields the image displayed in Figure 17. The original model was two layers with $10 \mathrm{ohm}-\mathrm{m}$ over $500 \mathrm{ohm}-\mathrm{m}$ with a layer thickness of 20 meters. The imaging scheme recovers this model almost exactly. This example serves as a self-consistency test for the algorithm.

As expected, the use of uniform halfspace coefficients does not provide equally good results. Figure 18, displaying the image for no offset between source and borehole, shows that distinct layering is not preserved. The contours basically follow the shape of the uniform halfspace current density diffusion process (Plate 1). A practiced eye will be able to discern that this pattern represents layering but none of the layering parameters are well defined. When the source is offset from the borehole an asymmetry is introduced into the image (Figure 19). The final word on the usefulness of using the uniform halfspace weighting coefficients is provided by adding a conductive she11 target as shown in Figure 20. Figure 21 compares the resulting image with that of Figure 19, and the images are practically identical. This holds true even when the layer resistivity contrast is reduced from 10:500 to 100:500, where the difference between the images was discernible but small. The resolution of the 2-D structure improves directly 


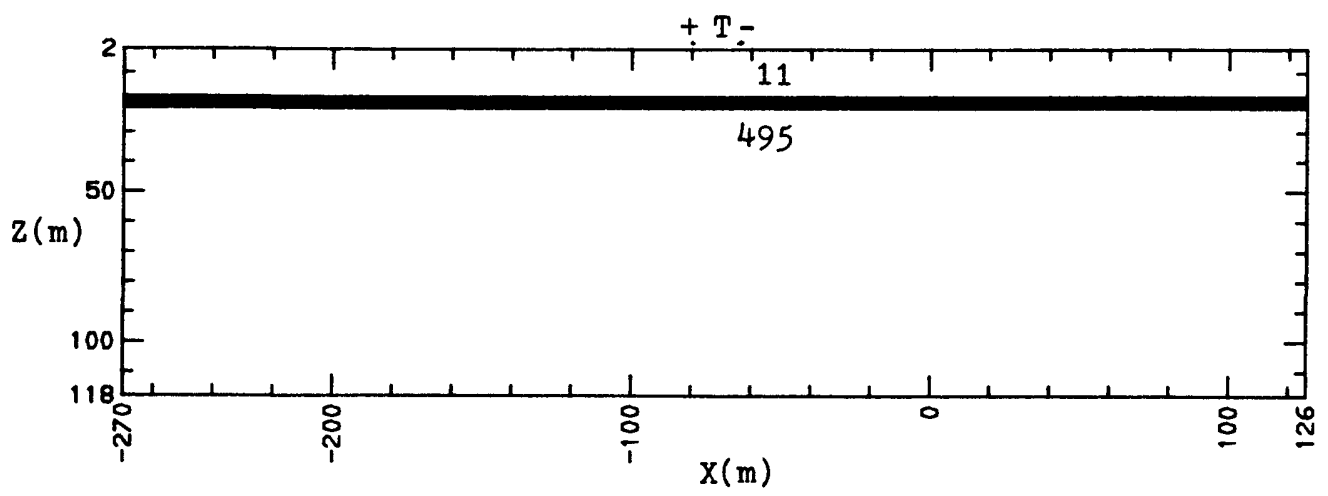

CR TEST, 72m OFFSET, 10 ALGORITHM

CONTOUR INTERVAL $=11,100,200,300,400,495$

Figure 17. Imaged apparent resistivity cross-section for a conductive overburden model and use of weighting coefficients for that model in the imaging process. Source - borehole separation is $72 \mathrm{~m}$.

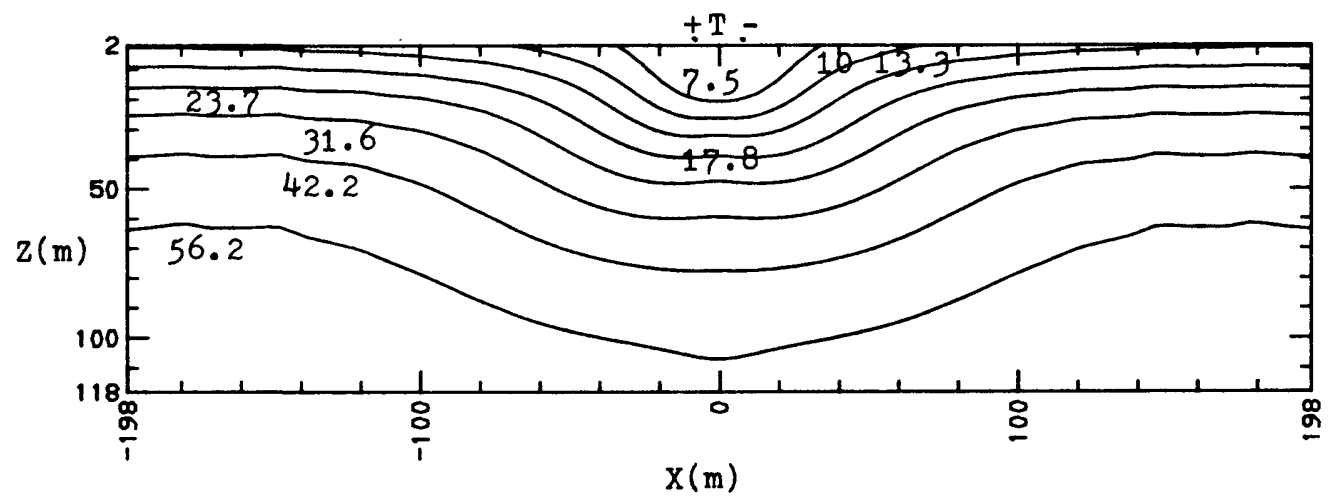

CR TEST, On OFFSET, HS ALGORITHM CONTOUR INTERVAL $=$ LDG $(8 /$ CYCLE)

Figure 18. Imaged apparent resistivity cross-section for the conductive overburden model and use of weighting coefficients for a $10 \mathrm{ohm}-\mathrm{m}$ uniform halfspace. There is no offset between source and borehole. 


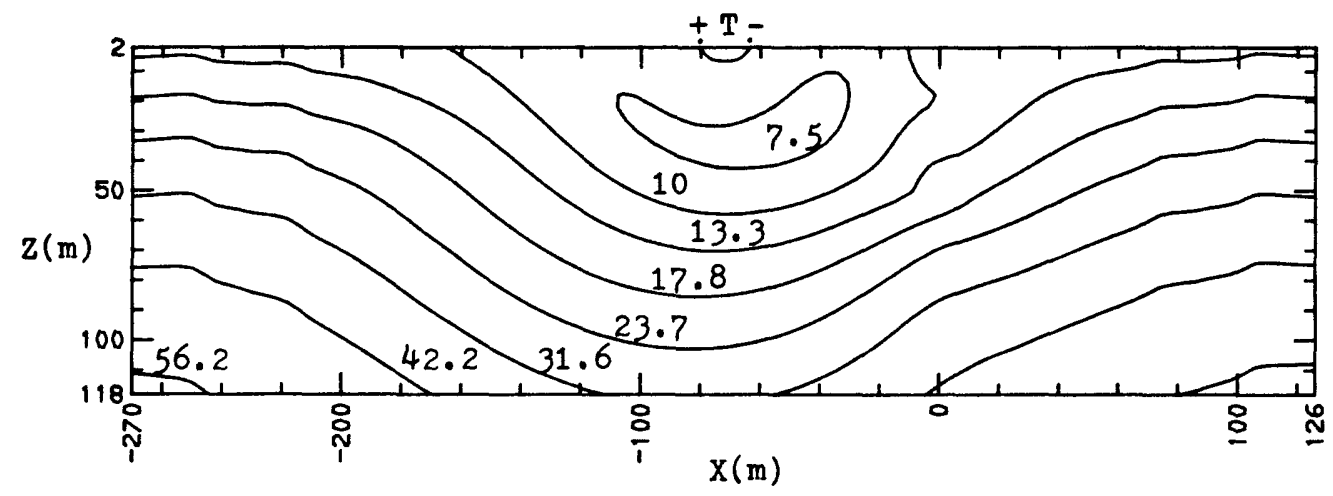

CR TEST, 72m OFFSET, HS ALGORITHM CONTOUR INTERVAL $=$ LOG $(8 /$ CYCLE)

Figure 19. Imaged apparent resistivity cross-section for the conductive overburden model and use of weighting coefficients for a $10 \mathrm{ohm}-\mathrm{m}$ uniform halfspace. There is now a $72 \mathrm{~m}$ offset between source and borehole.

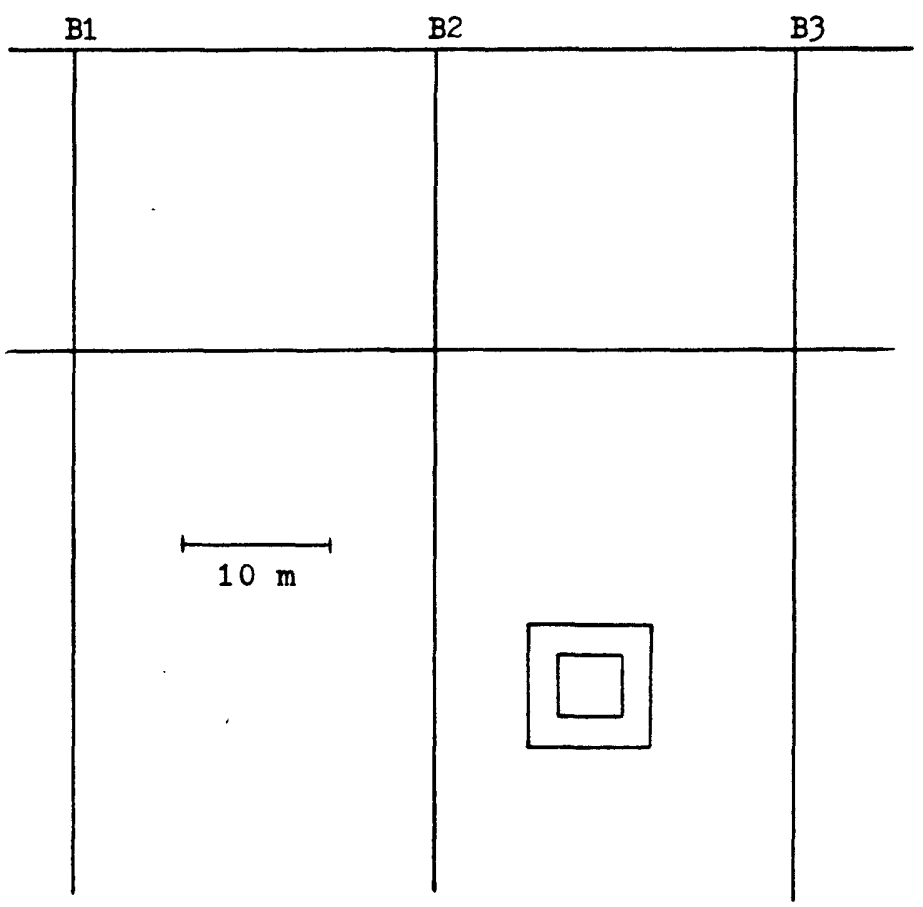

Figure 20. Mode1 of a tunnel with conductive shell beneath conductive overburden. The overburden is $10 \mathrm{ohm}-\mathrm{m}$, the basement is $500 \mathrm{ohm}-\mathrm{m}$ and the shell is $150 \mathrm{ohm}-\mathrm{m}$. The overburden thickness is $20 \mathrm{~m}$. All other dimensions are the same as Figure 11 . 


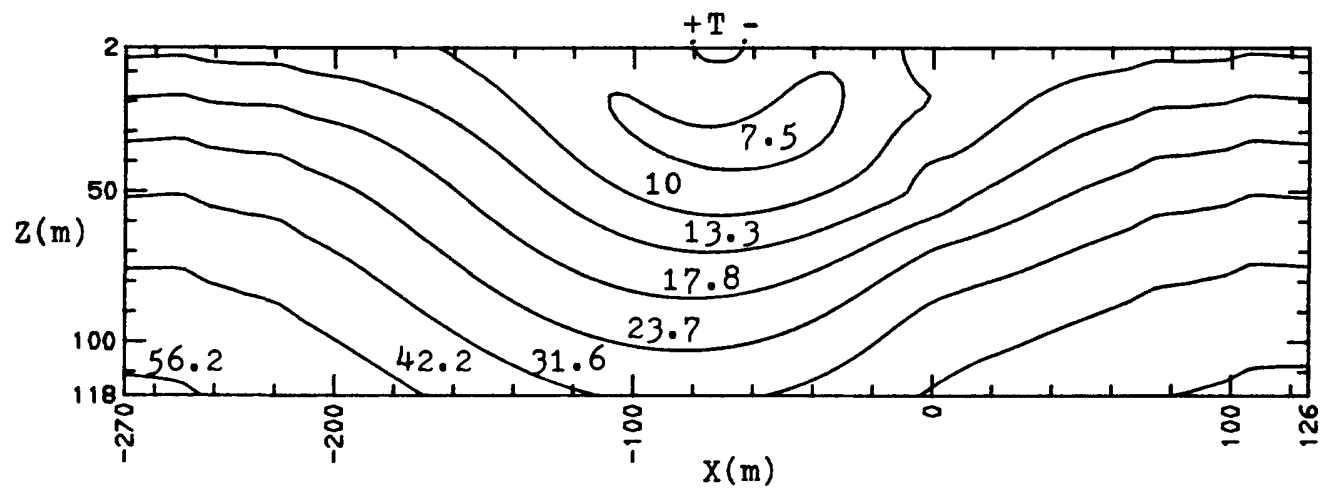

(a)

TUNNEL $* 7$, SRC 2. BHOLE 2 CONTOUR INTERVAL $=$ LOG $(8 /$ CYCLE $)$

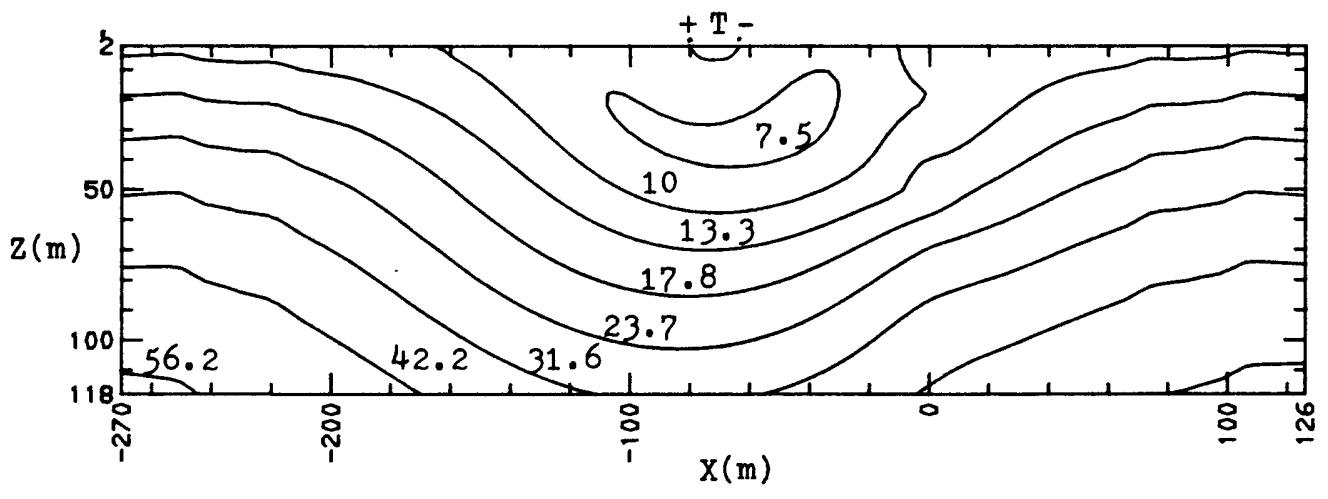

(b)

CR TEST,72m OFFSET. HS ALCORITHM CONTOUR INTERVAL $=$ LOG $(8 / \mathrm{CYCLE})$

Figure 21. (a) Imaged apparent resistivity cross-section for the model of Figure 20. Weighting coefficients for a uniform halfspace of 10 ohm-m are used. There is a $72 \mathrm{~m}$ offset between source and borehole. (b) Repeat of Figure 19, the image resulting when no tunnel is present. 
with the estimate of the layered host rock structure. Effort should always be made to obtain a good estimate of this layered structure.

A profile of six images, utilizing the weighting coefficients for the layered model presented in Figure 20, is displayed in Figure 22. The two layer structure is entirely recovered and the tunnel is quite distinct. The general features are very similar to those seen in Figure 8 . Here again the images in (c), (d), and (e) display false resistive anomalies. Conductive anomaly estimates for these images can be created by the same procedure described previously.

Creating a composite image for these six images is also achieved as previously described. This composite image is displayed in Figure 23. The same positional resolution is achieved as for the unfform host rock case (Figure 12). Repeating the process for another two-layer model, in which the only difference is that the first layer resistivity is now $100 \mathrm{ohm}-\mathrm{m}$, yields the composite image of Figure 24. Upon comparing Figures 23 and 24 , note that the imaged anomaly is stronger under the more weakly conducting overburden, illustrating the masking effect of high1y conductive overburden. 


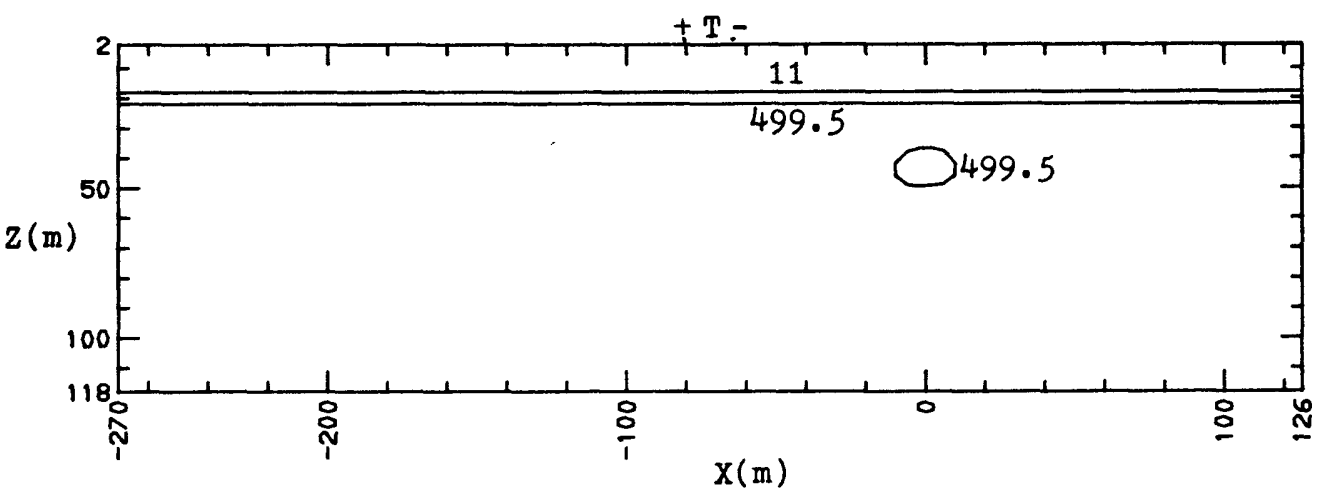

(a)

TUNNEL $\% 7$, SRC 1, BHOLE 1 CONTOUR INTERVAL $=11,499.5$

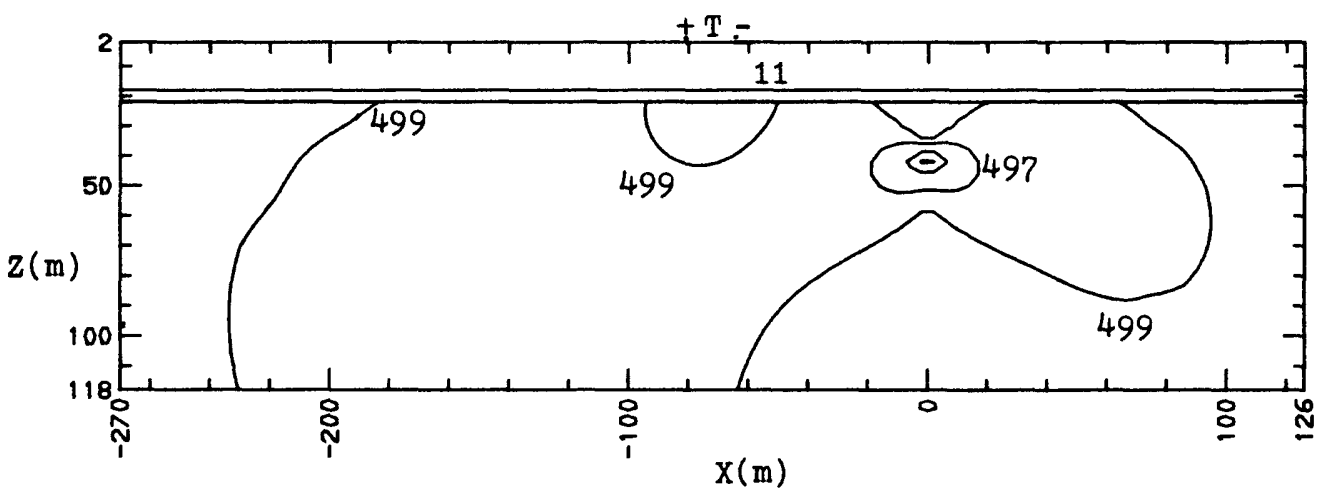

(b)

TUNNEL $\# 7$, SRC 2, BHOLE 2 CONTOUR INTERVAL $=11,490,494,497,499$

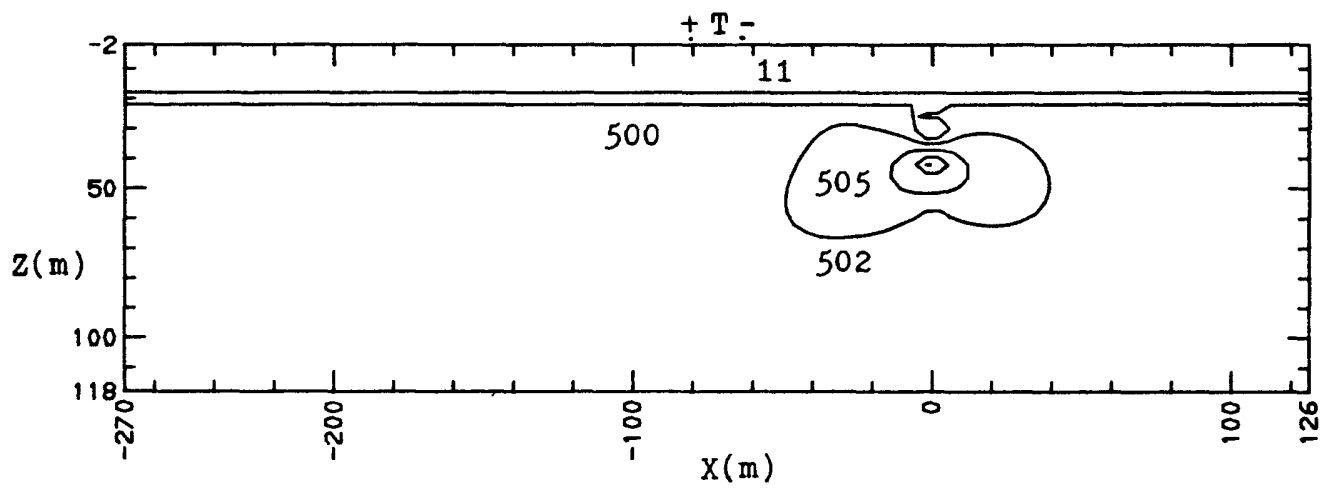

(c)

TUNNEL \#7, SRC 3. BHOLE 3

CONTOUR INTERVAL $=11,500,502,505,509,514$

Figure 22. Imaged apparent resistivity cross-section for the model of Figure 20 for six different source - borehole combinations, all with a separation of $72 \mathrm{~m}$. The correct weighting coefficients for the layered reference model are used. Images for (a) source $\mathrm{Sl}$ and borehole B1, (b) source S2 and borehole B2, and (c) source S3 and borehole B3. 


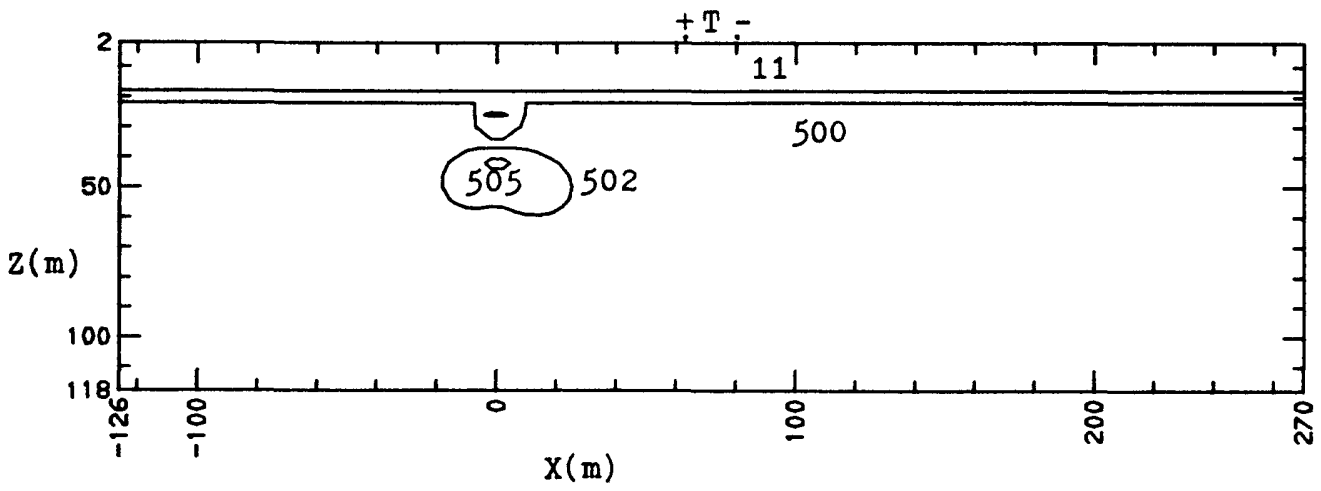

(d)

TLNNEL 7. SRC 7. BHOLE 1

CONTOUR INTERVAL $=11,500,502,505$

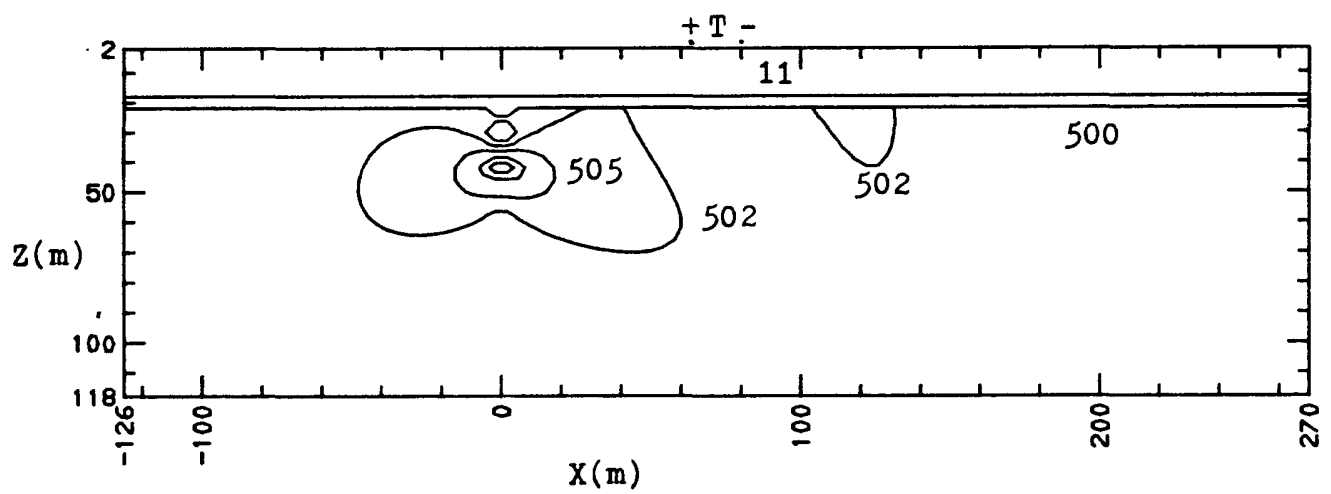

(e)

TUNNEL $\% 7$, SRC 8, BHOLE 2

CONTOUR INTERVAL $=11,500,502,505,509.514$

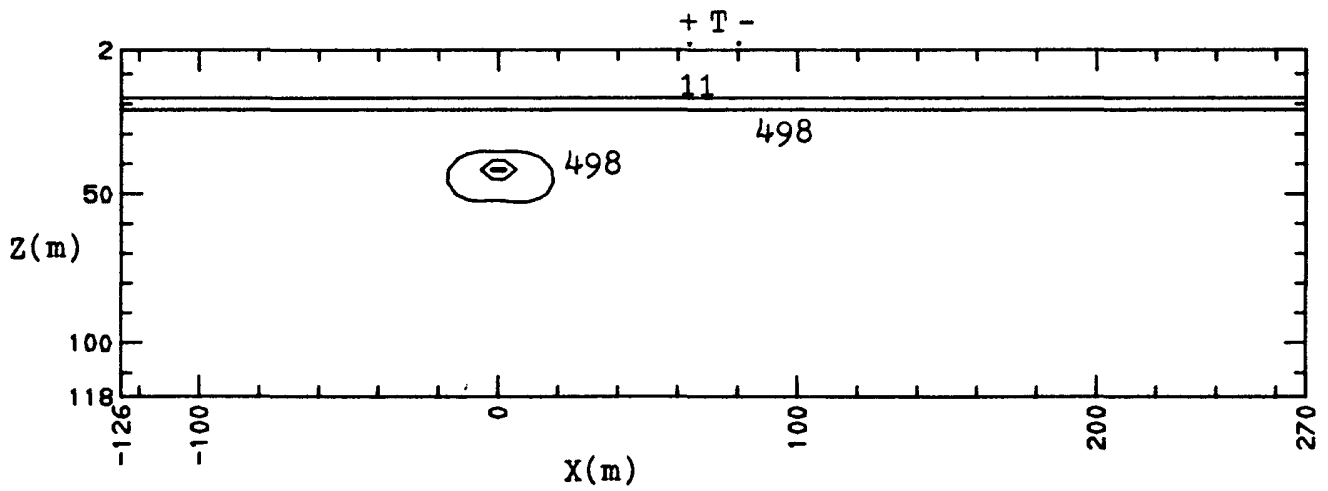

(f)

TUNNEL $\% 7$, SRC 9, BHOLE 3 CONTOUR INTERVAL $=11,494,496,498$

Figure 22. (continued) Images for (d) source S7 and borehole B1, (e) source S8 and borehole B2, and (f) source S9 and borehole B3. 


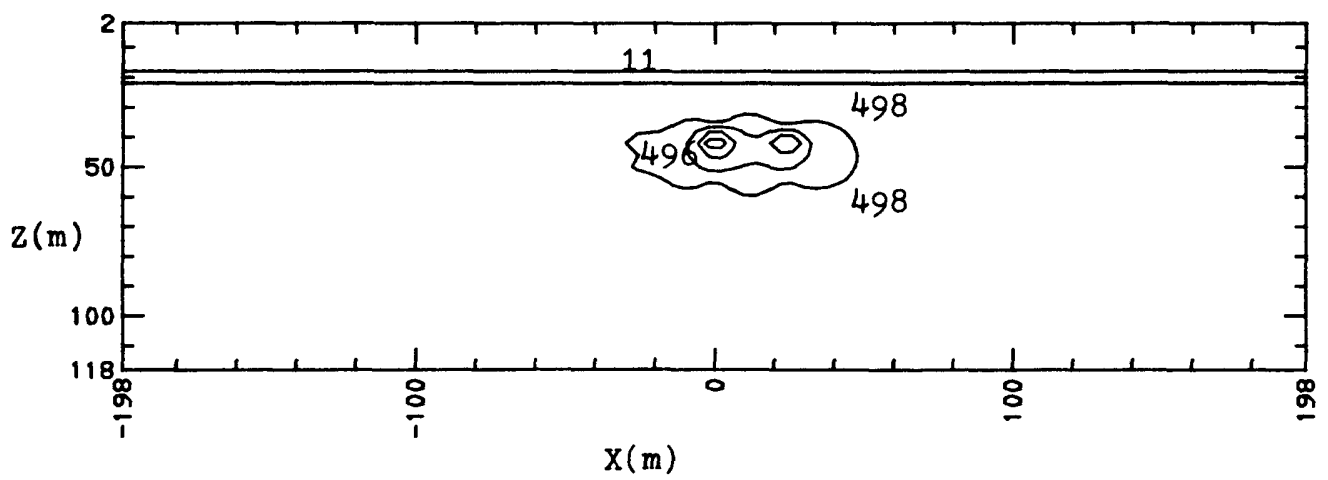

TUNNEL $* 7$ COMPOSITE IMAGE

CONTOUR INTERVAL $=11.490,494,496.498$

Figure 23. Composite apparent resistivity image incorporating all six data sets presented in Figure 22. Borehole B2 lies at position $X=0$ $\mathrm{m}$. The true lateral position of the center of the target is at $\mathrm{X}=6 \mathrm{~m}$.

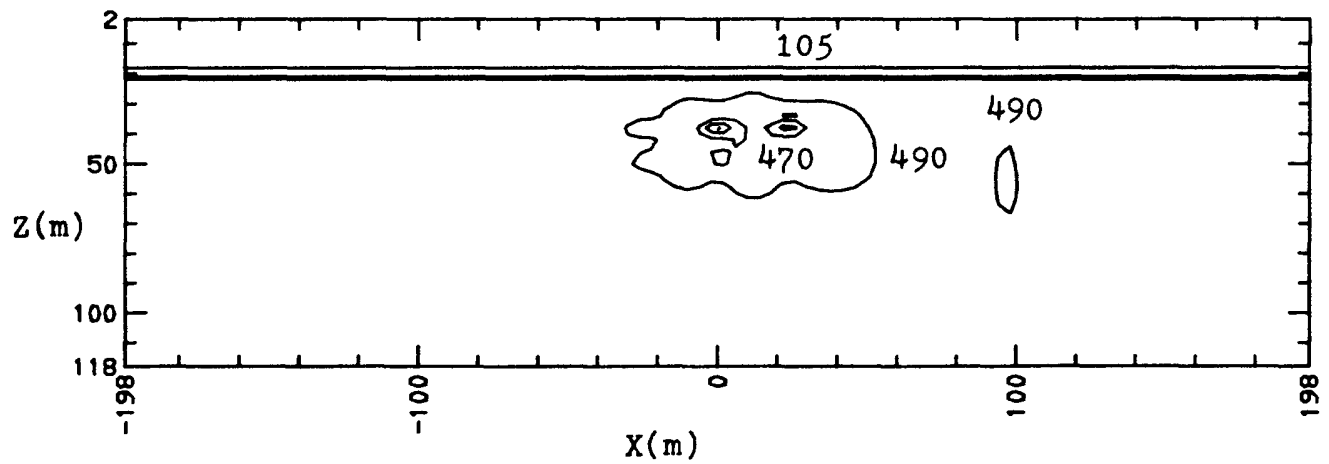

TUNNEL 10 COMPOSITE IMAGE

CONTOUR INTERVAL $=105,400,440,470,490$

Figure 24. Composite apparent resistivity image for the model of Figure 20 with the overburden resistivity changed to $100 \mathrm{ohm}-\mathrm{m}$. 


\section{Discussion}

The imaging procedure, as described, works for the simple models and theoretical testing shown. However, it is still just a prototype. Significant improvements can be accomplished as workable ideas are discovered for extracting more information from the complicated nature of the EM diffusion process. As it presently stands the imaging algorithm has weaknesses. A number of alternative mechanisms for many of these weak points await testing. The research to date has produced a viable, though awkward, algorithm. The basic approach appears to be sound. Substantial research remains to refine the process into production environment capability. The imaging algorithm is currently tailored to surface source borehole receiver geometries. Adaptation to surface receiver arrays requires only minor modifications. There are several stages of computer calculations performed but the computer time used is modest. The procedure is primarily data storage intensive; a large disk space is essential. With the more recent generation of personal computers, however, it is reasonable to expect this imaging procedure to work well in the personal computer environment. It should work extremely well in the new and more robust personal workstation environment. 
PART V: SUMMARY, CONCLUSIONS, AND RECOMMENDATIONS

Summary

This report presents the concept, prototype algorithms and testing of a new method of TEM processing to produce a subsurface resistivity image for application to tunnel detection. The imaging concept is based on the fact that variable contributions to all measured magnetic field (or its time-rateof-change) locations come from everywhere in the subsurface at all times. It is possible to rapidly calculate, on a grid, these contributions from calculated subsurface current densities for any layered resistivity structure using Biot-Savart's Law. These calculated contributions (a function of subsurface position, receiver position and time) are used as weighting coefficients to extract fractions of measured magnetic field values reflecting the influence of some 2-D or 3-D structure within the layered host. Al1 of these fractions are summed for each subsurface element to yield, when normalized by a similar sum for synthetic data reflecting only the layered host and multiplied by the conductivity assumed for that element, an estimate of the conductivity of that element (an apparent conductivity). Contouring the estimates for all subsurface elements provides an image of the 2-D subsurface resistivity distribution.

The feasibility of using this TEM imaging technique for tunnel detection was studied for the cases of: tunnel with conductive shell vs. tunnel with no conductive shell, uniform halfspace host vs. two-layer host, and loop source vs. grounded-wire source. Results are presented as contour plots of the 2-D resistivity distribution estimated by the imaging procedure. 


\section{Conclusions}

The prototype TEM imaging procedure described herein is successful at generating an effective subsurface resistivity image for the models tested and the surface source-borehole receiver geometry utilized. The results clearly indicate that, to obtain quality image resolution of the 2-D structure, a good estimate of the 1-D (1ayered) host structure is needed. The resulting resistivity image presents a "fuzzy", but reasonable, estimate of the true 2-D structure. Approximate location of the structure is provided and further positional detail can possibly be derived from the shape of the imaged resistivity anomaly. The TEM imaging concept has considerable promise as a useful 2-D and 3-D structural interpretation tool in tunnel detection and many other geophysical mapping applications.

Being based on the induction process, TEM methods are primarily suited for detection of conductive structures. The model study clearly reaffirms this in that a tunnel surrounded by a conductive shell creates a strong anomaly and a tunnel not surrounded by such a shell creates only a weak anomaly. The results suggest that a conductive shell tunnel structure would be detectable several tens of meters away from the borehole. With no conductive she11, however, a tunnel would likely only be detectable if it were within one or two tunnel diameters of the borehole. The influence of geologic noise on this resolution has not been determined.

The TEM survey design tested for tunnel detection consists of a profile of evenly spaced receivers down a borehole and a f xed source-borehole separation with the whole array rolling along. This requires a profile of evenly spaced boreholes and multiple sources at the same spacing. The pattern of data acquisition is very redundant and similar to standard seismic reflection data acquisition. 
The present TEM imaging algorithm still does not utilize all of the information available through use of the 2-D nature of the EM field and contribution pattern. Parts of the algorithm are definitely awkward. Furthermore, the imaged resistivity anomaly is on 1y approximately one third of the maximum EM field anomaly seen in the data. More resolution is definitely available. Many variations are possible for the mechanisms to calculate weighting coefficients and to stack the data into an image. More research on these mechanisms is a high priority. Also, the effectiveness of the TEM imaging algorithm in the presence of geologic noise needs to be addressed. What are the method's limitations?

Finally, a field test needs to be performed to assess the method's performance using real data. It is likely that a number of modifications and improvements in the algorithm would result from having this data. A field test would further allow the U.S. Army to assess the effectiveness of TEM methods/imaging for tunnel detection in specific geologic environments and as a general reconnaissance tool. 


\section{References}

Barnett, C. T., 1984, Simple inversion of time-domain electromagnetic data: Geophysics, v. 49, p. 925-933.

Dhar, R. L., 1972, Studies on investigation characteristics of electromagnetic and electrical prospecting systems, and interpretation of vertical gravity and magnetic profiles in boreholes and shafts: Ph.D. Thesis, Banares Hindu University, India.

Dines, K. A., and Lytle, R. J., 1981, Analysis of electrical conductivity imaging: Geophysics, v. 46, p. 1025-1036.

Dyck, A. V., and West, G. F., 1984, The role of simple computer models in interpretations of wide-band, dri11-hole electromagnetic surveys in mineral exploration: Geophysics, v. 49, p. 957-980.

Kauahikaua, J. P., 1982, The subsurface resistivity structure of Kilauea Volcano, Hawaii: Ph.D. dissertation, University of Hawaii.

Lee, S., McMechan, G. A., and Aiken, C. L. V., 1987, Phase-field imaging: the electromagnetic equivalent of seismic migration: Geophysics, v. 52, p. 678-693.

Levy, S., O1denburg, D., and Wang, J., 1988, Subsurface imaging using magnetotelluric data: Geophysics, v. 53, p. 104-117.

Macnae, J., and Lamontagne, Y., 1987, Imaging quasi-layered conductive structures by simple processing of transient electromagnetic data: Geophysics, v. 52, p. 545-554.

Nabighian, M. N., 1979, Quasi-static transient response of a conducting halfspace - An approximate representation: Geophysics, v. 44, p. 17001705. 
Nabighian, M. N., ed., 1984, Time-Domain Electromagnetic Methods of Exploration: Geophysics - Special Issue, v. 49, p. 849-1029.

Newman, G. A., Hohmann, G. W., and Anderson, W. L., 1986, Transient electromagnetic response of a three-dimensional body in a layered earth: Geophysics, v. 51, p. 1608-1627.

Nekut, A. G., 1987, Direct inversion of time-domain electromagnetic data, Geophysics, v. 52, p, 1431-1435.

Newman, G. A., Anderson, W. L., and Hohmann, G. W., 1987, Interpretation of transient electromagnetic soundings over three-dimensional structures for the central - loop configuration: Geophys. J. R. Astr. Soc., v. 89, p. 889-914.

Oristaglio, M. L., and Hohmann, G. W., 1984, Diffusion of electromagnetic fields into a two-dimensional earth: A finite-difference approach: Geophysics, v. 49, p. 870-894.

Patra, H. P., and Ma1lick, K., 1980, Geosounding Principles: Volume 2: TimeVarying Geoelectric Soundings: Elsevier Science Publishing Company, New York.

Roy, A., and Dhar, R. L., 1970, Relative contribution to signal by ground elements in two-coil induction logging system: Geophysical Prospecting, v. 18, p. $389-404$.

Sidorov, V. A., and Gubatenko, V. P., 1974, on the resolution of electromagnetic prospecting by the build-up method: Physics of the Solid Earth (English ed.), no. 3, p. 173-176. 
Plate 1. Color contour cross-sections of the current density within a 100 ohm-m uniform halfspace, as a function of time, created by a loop source of dimensions $200 \mathrm{~m}$ by $1000 \mathrm{~m}$ (see Figure 2). The physical dimensions of the plots are $5000 \mathrm{~m}$ by $1500 \mathrm{~m}$ ( 100 by 30 pixels). Current density is in units of $\mathrm{A} / \mathrm{m}^{2}$.

$$
* E-8
$$

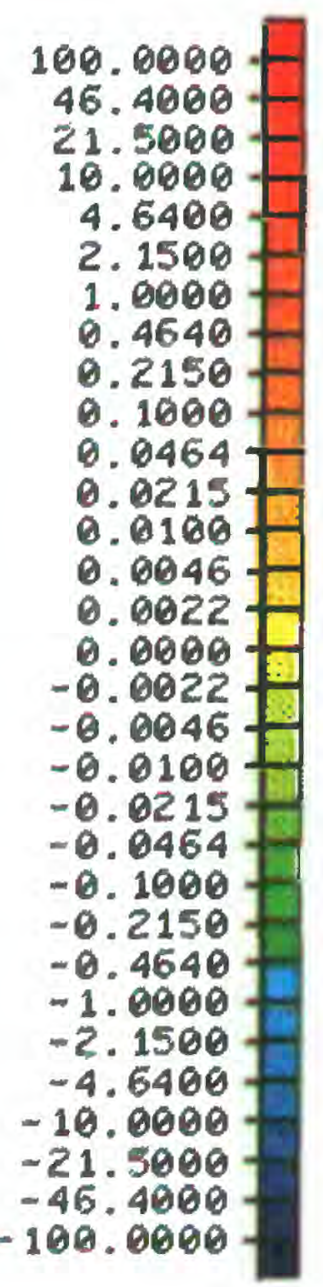

46 
HALFSPACE JY AT $T=.01 \mathrm{MS}$

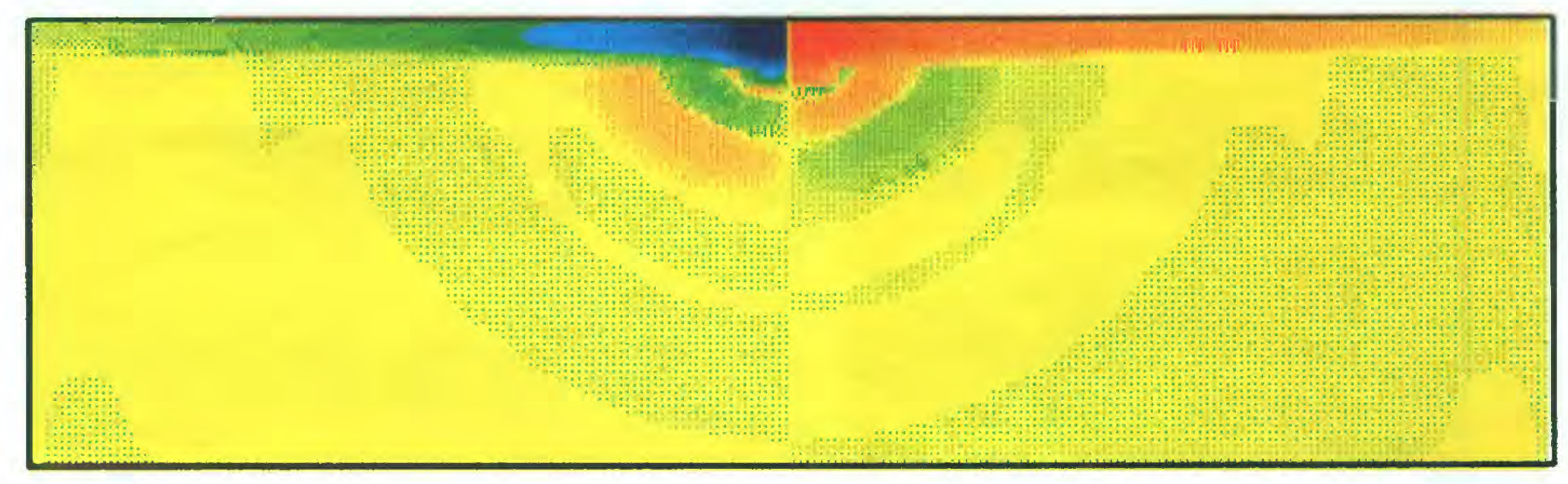

HALFSPACE JY AT T $=.031 \mathrm{MS}$

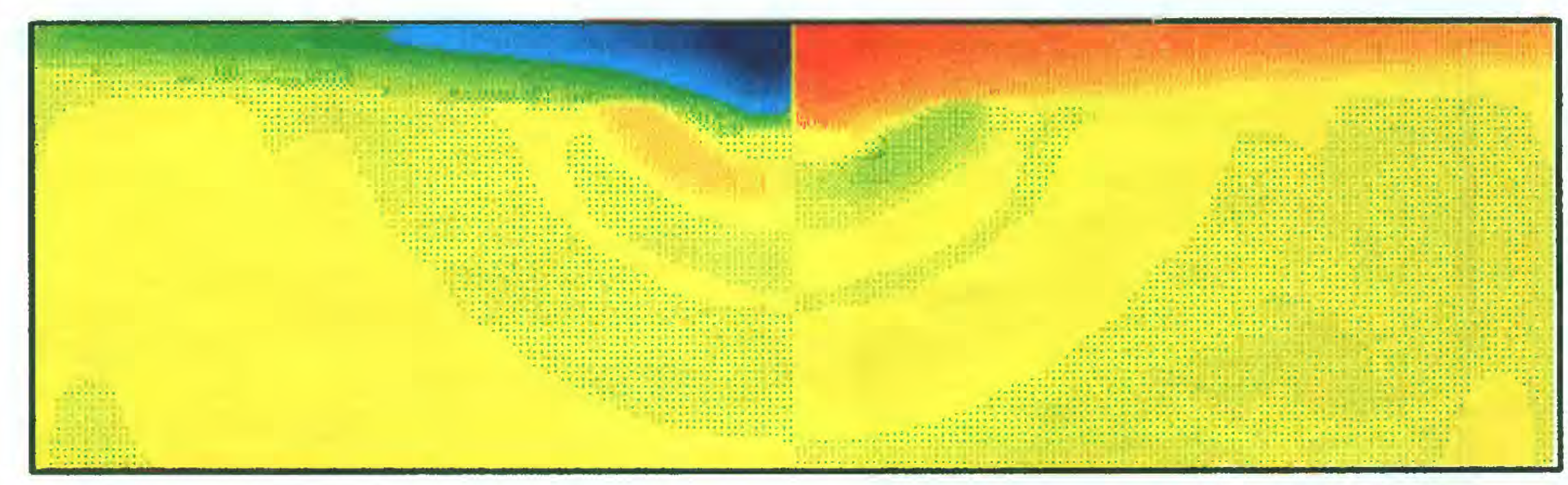

HALFSPACE IY AT $T=.1 \mathrm{MS}$

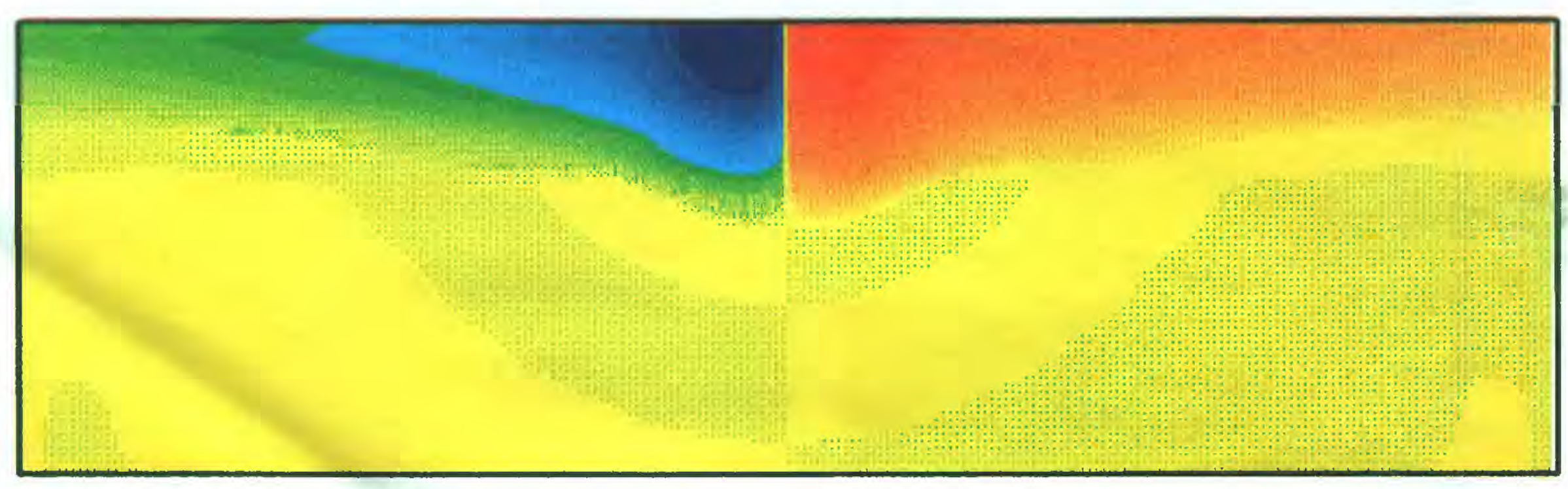

HALFSPACE JY AT $T=.31 \mathrm{MS}$

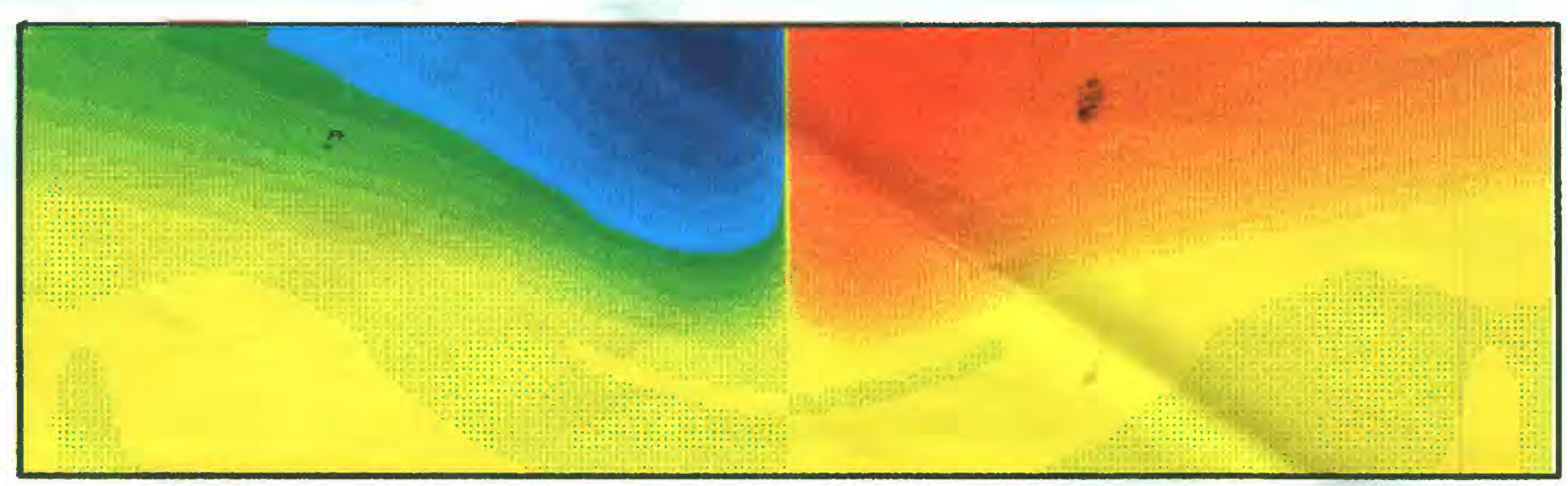


HALFSPACE UY AT $T=1 \mathrm{MS}$

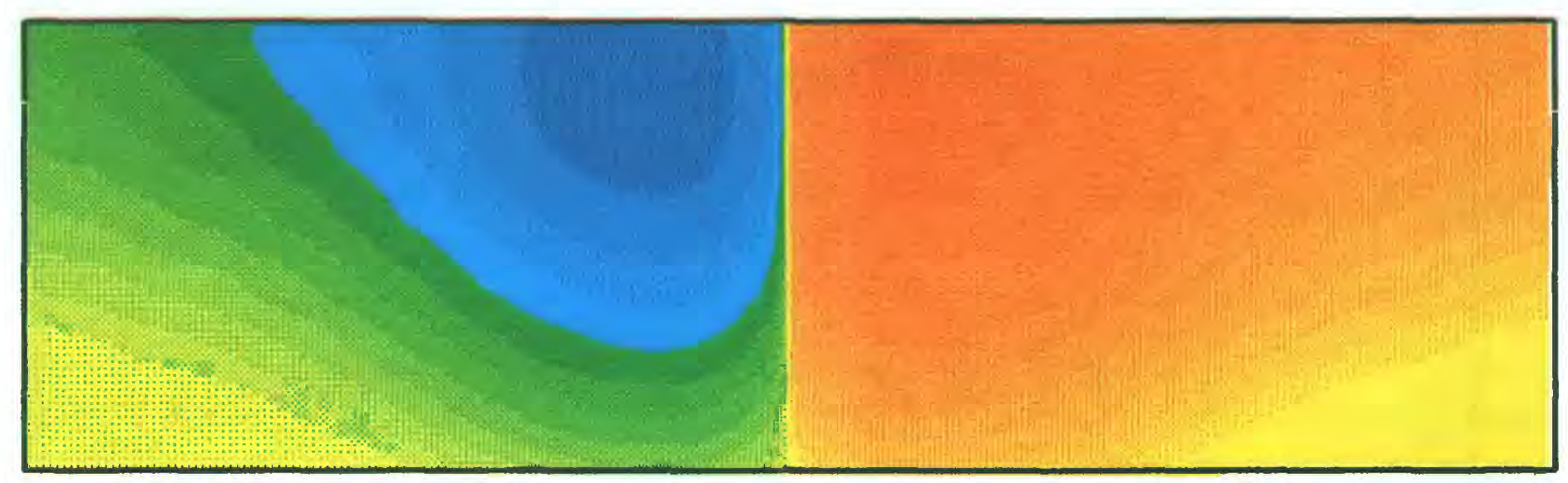

HALFSPACE JY AT $T=3.1 \mathrm{MS}$

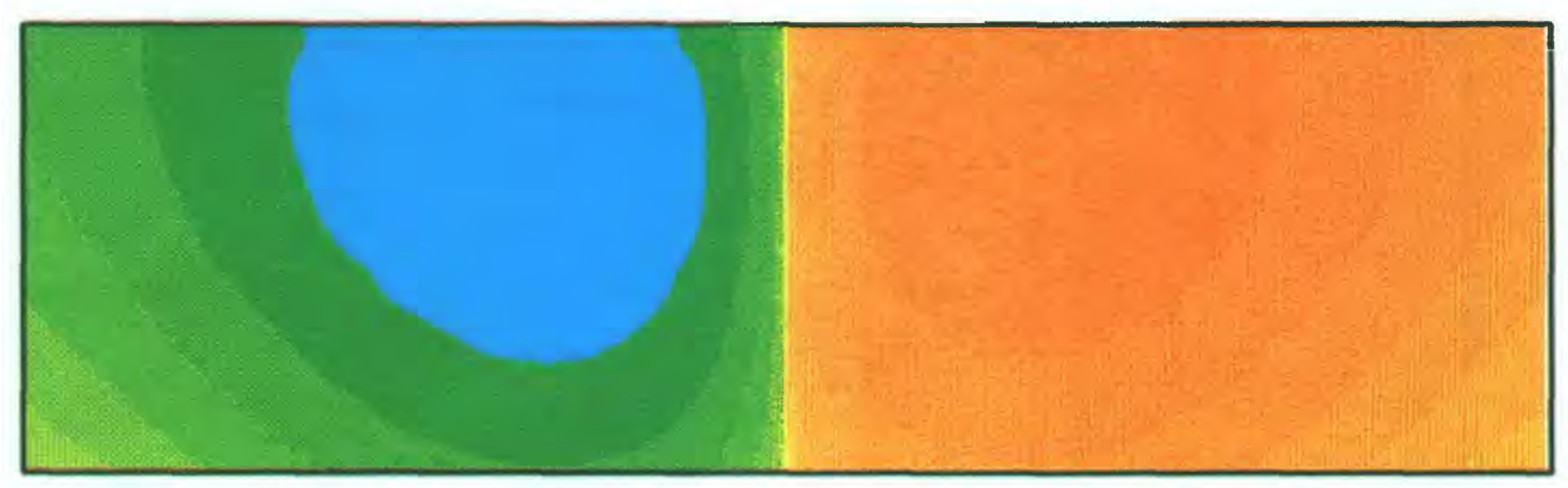

HALFSPACE UY AT T $=10 \mathrm{MS}$

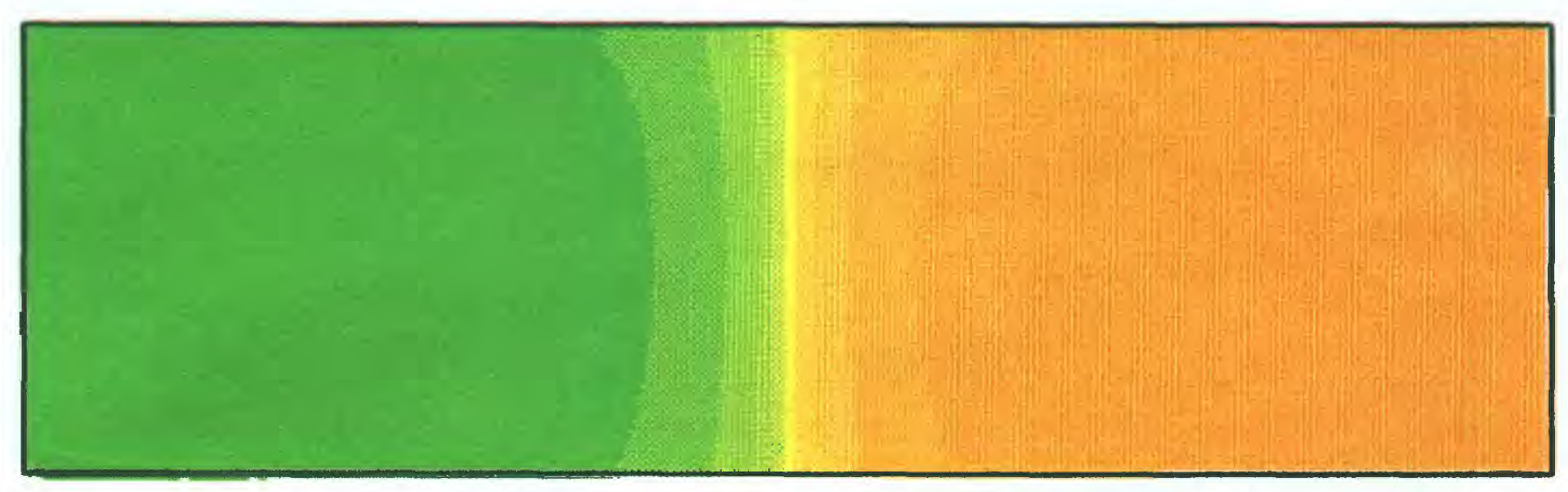

HALFSPACE JY AT T $=31 \mathrm{MS}$

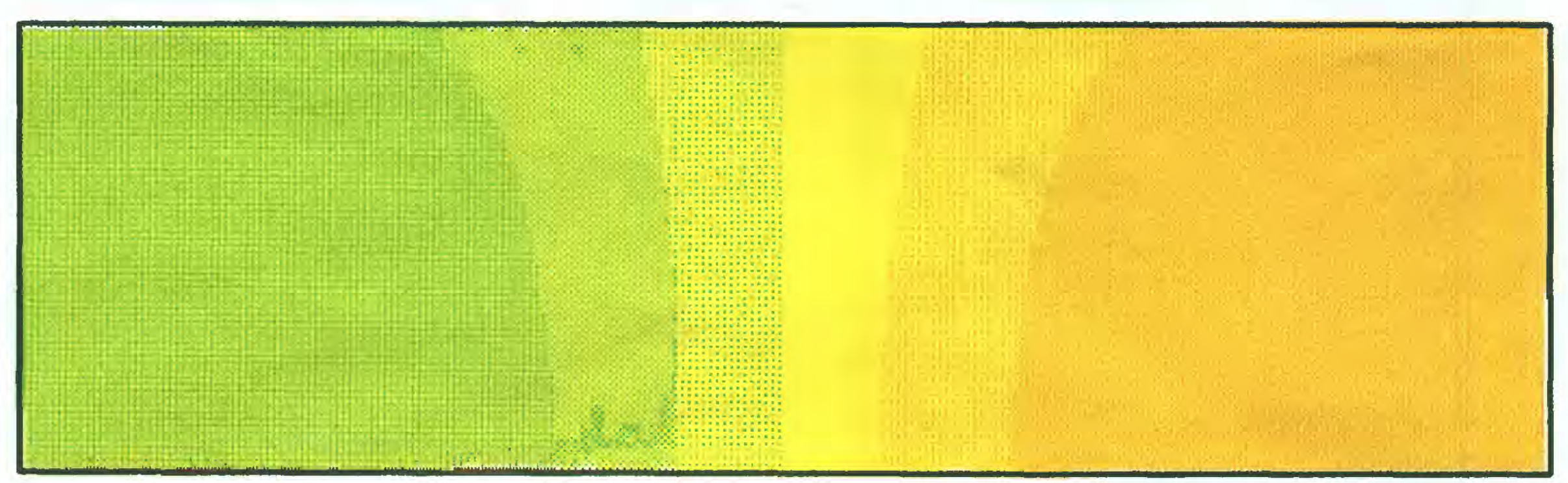


Plate 2. Color cross-sections of the relative contribution, $h_{z} / H_{z}$, of the current densities displayed in Plate 1 to the $z$-component of the magnetic field at position $X=900 \mathrm{~m}, Z=525 \mathrm{~m}$.

$* E-11$

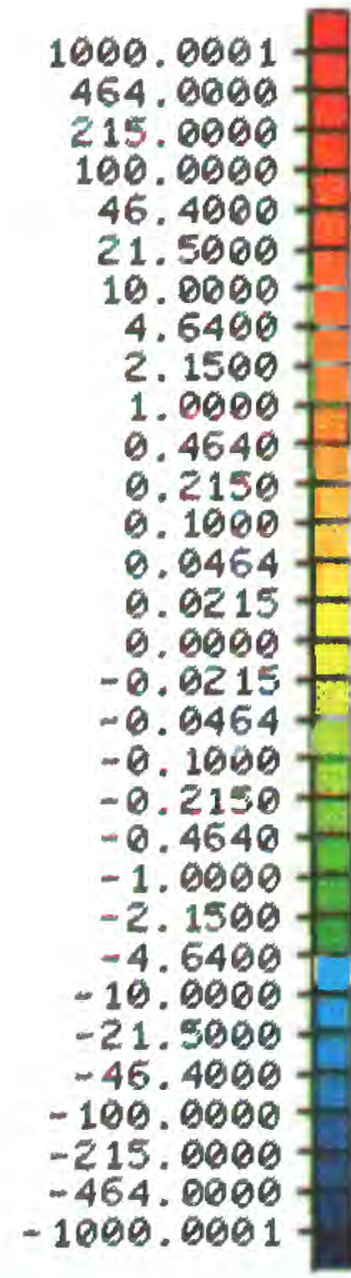

49 
HALFSPACE HZ CONTRIB. AT $T=.01 \mathrm{MS}$

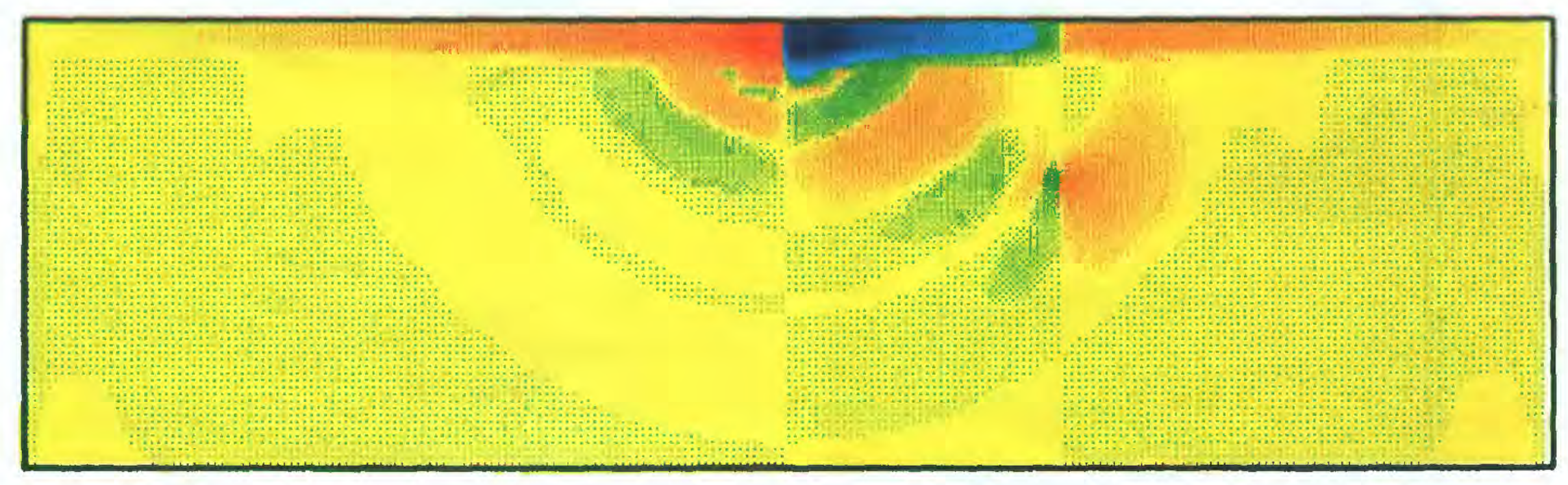

HALFSPACE HZ CONTRIB. AT $T=.031 \mathrm{MS}$

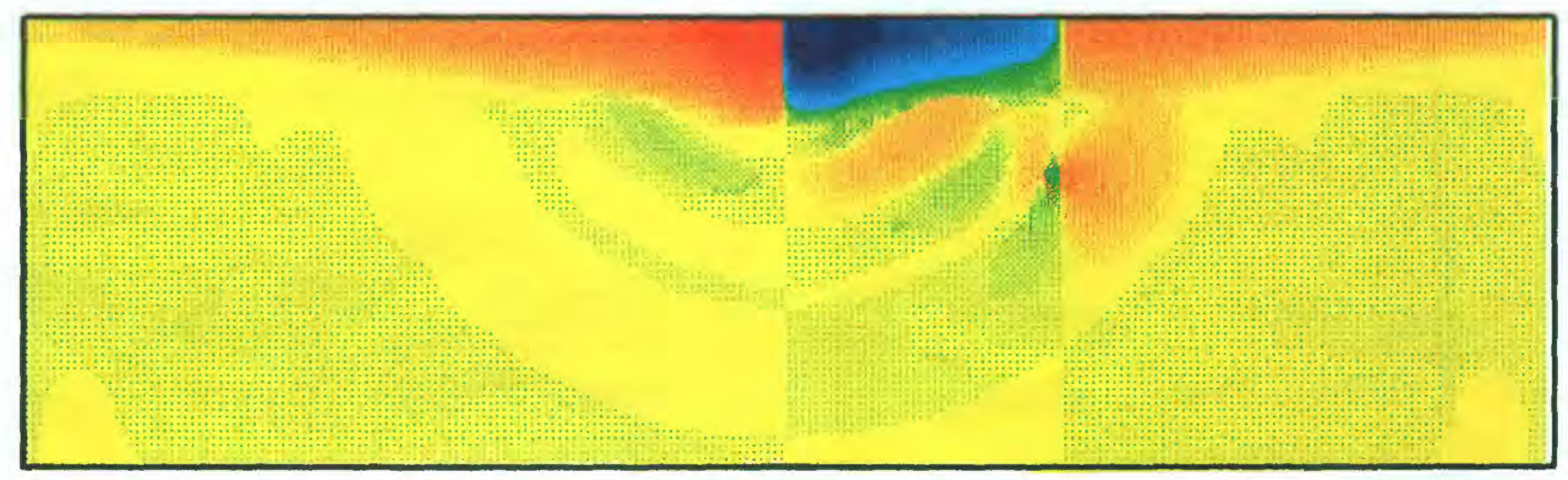

HALFSPACE HZ CONTRIB. AT T $=.1 \mathrm{MS}$

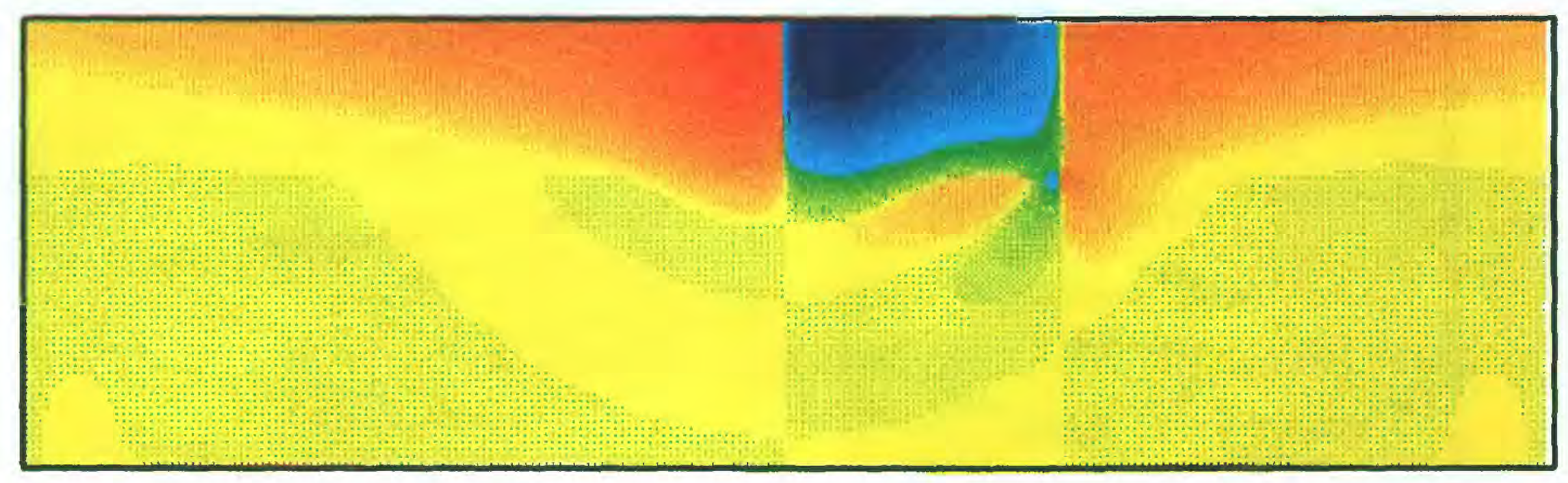

HALFSPACE HZ CONTRIB. AT $T=.31 \mathrm{MS}$

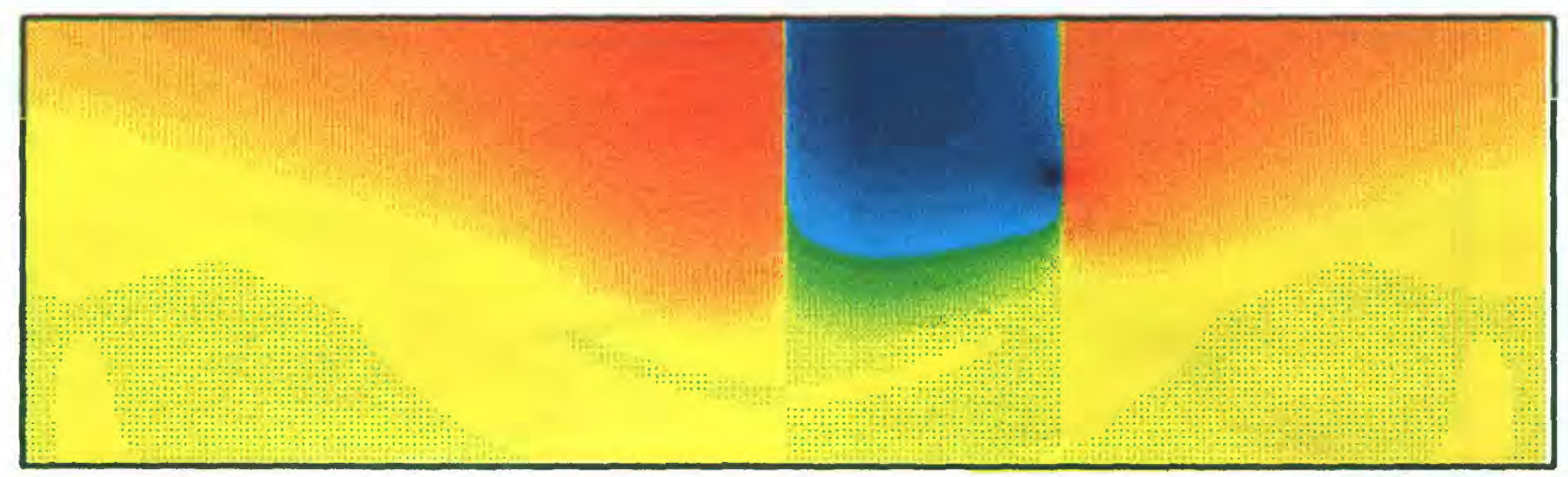


HALFSPACE $H Z$ CONTRIB. AT T $=1 \mathrm{MS}$

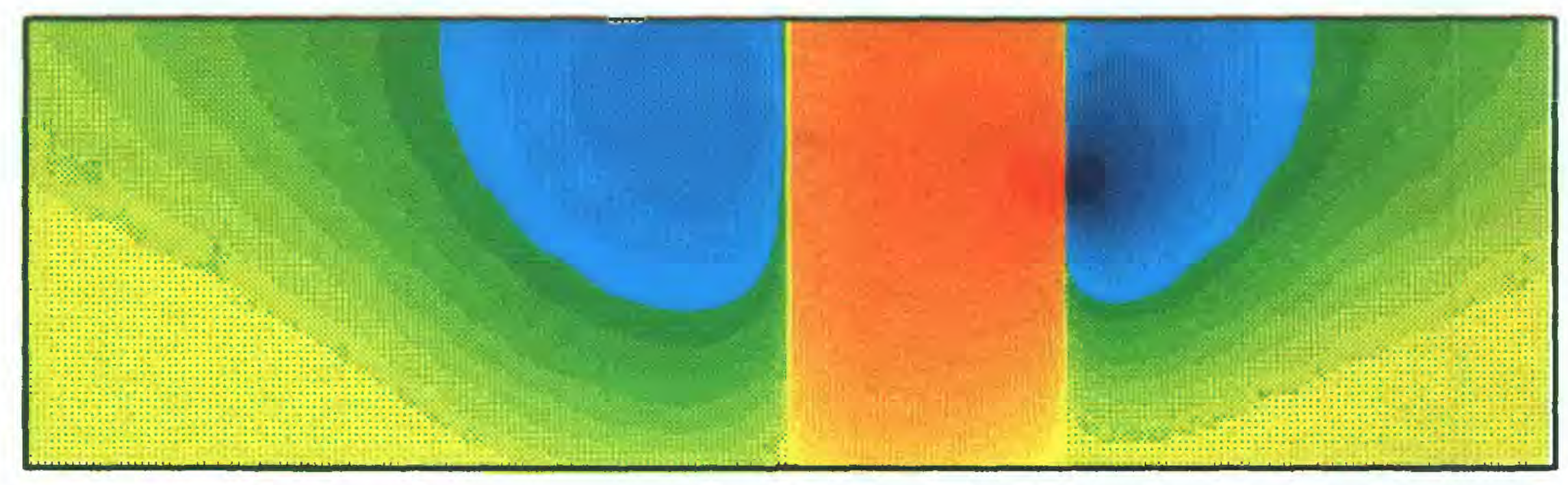

HALFSPACE HZ CONTRIB. AT T $=3.1 \mathrm{MS}$

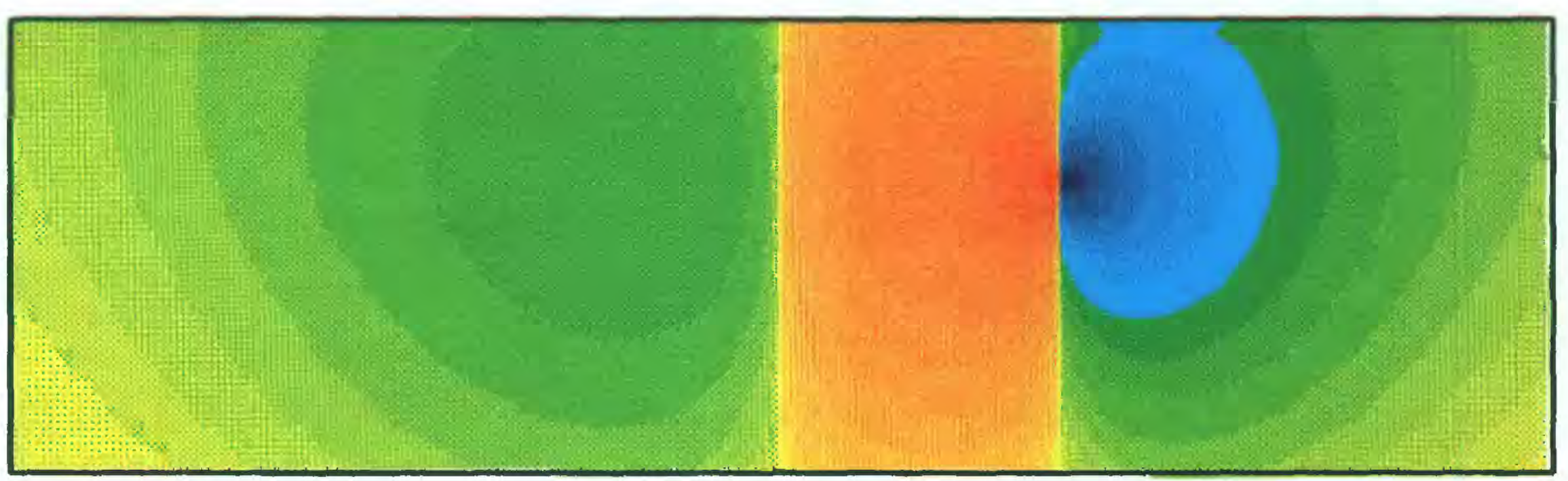

HALFSPACE $\mathrm{HZ}$ CONTRIB. AT $T=10 \mathrm{MS}$

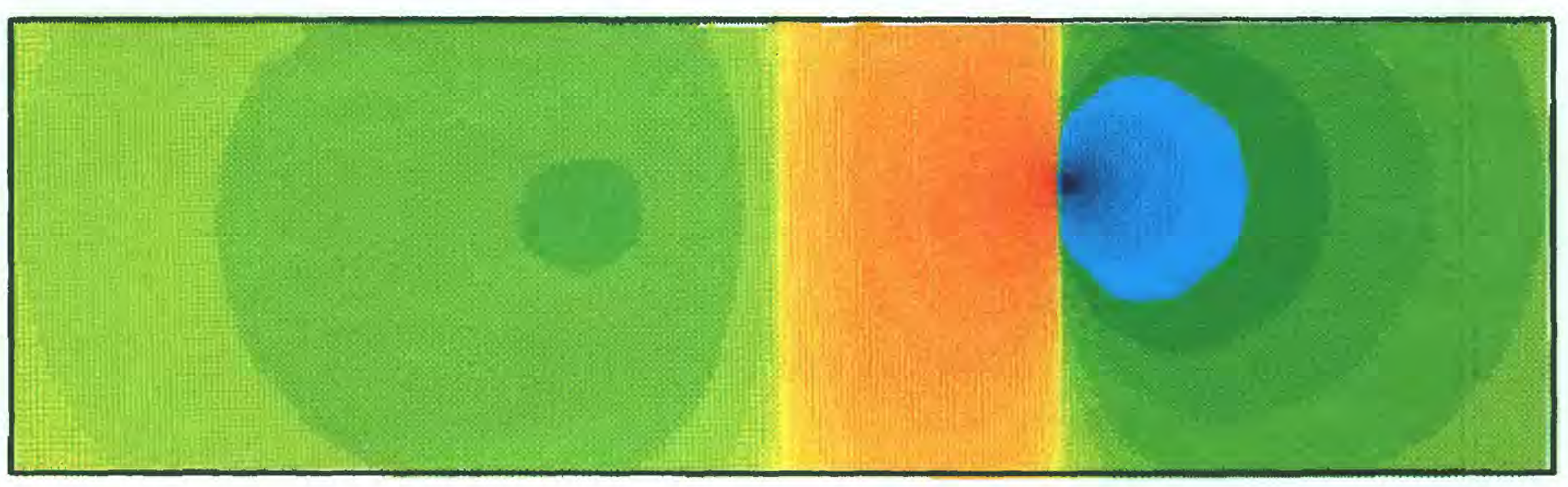

HALFSPACE HZ CONTRIB. AT $T=31 \mathrm{MS}$

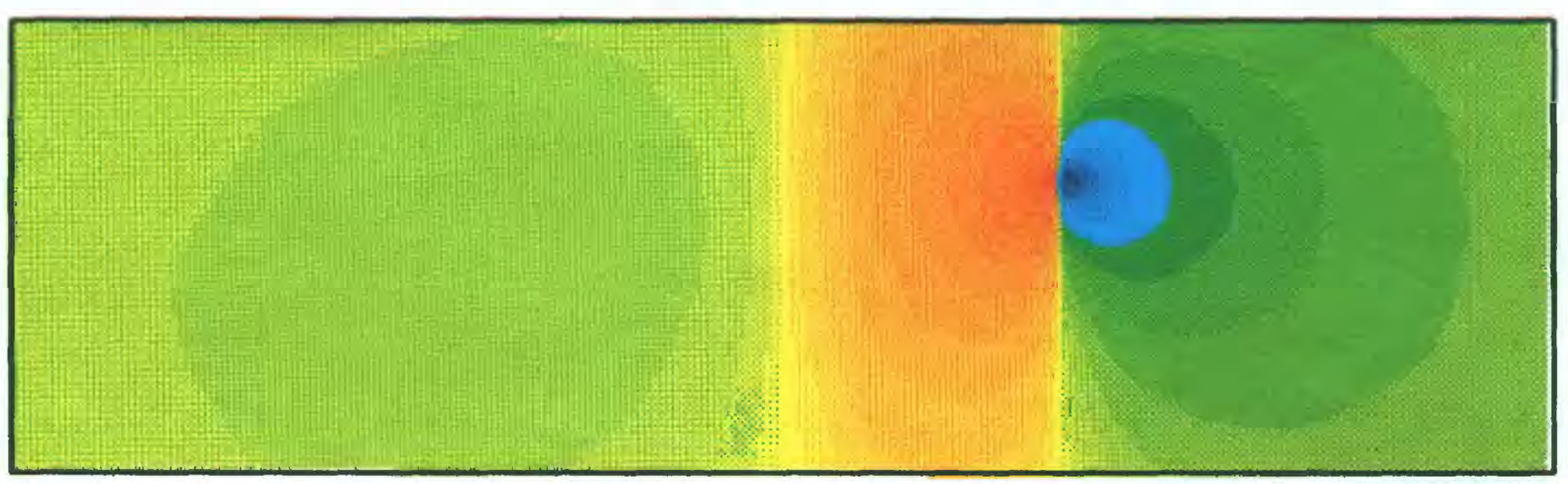


Plate 3. Color cross-sections of the relative contribution, $h_{z} / H_{z}$, of current densities for a conductive overburden model to the $z-$ component of the magnetic field at position $X=900 \mathrm{~m}, \mathrm{Z}=525 \mathrm{~m}$.

$* E-11$

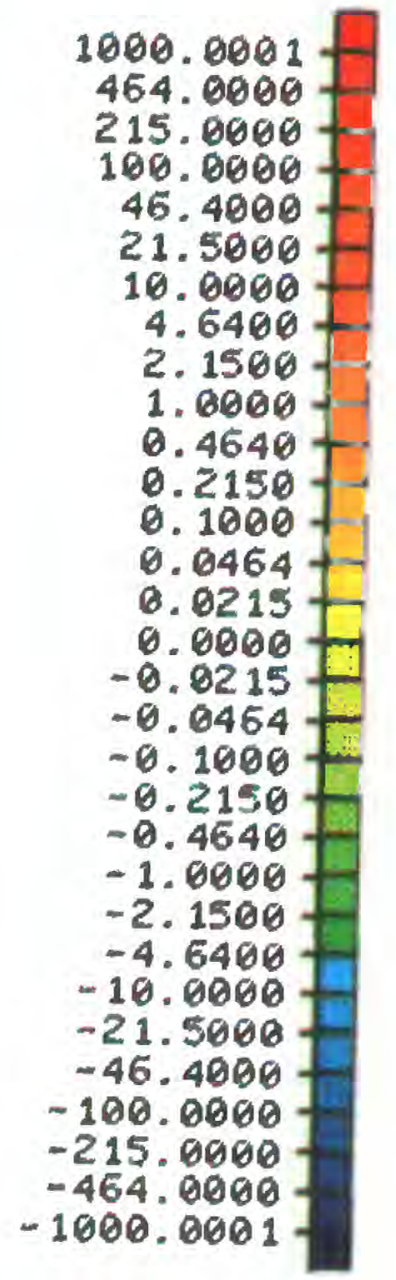


OVERBURDEN HZ CONTRIB. AT T $=.01 \mathrm{MS}$

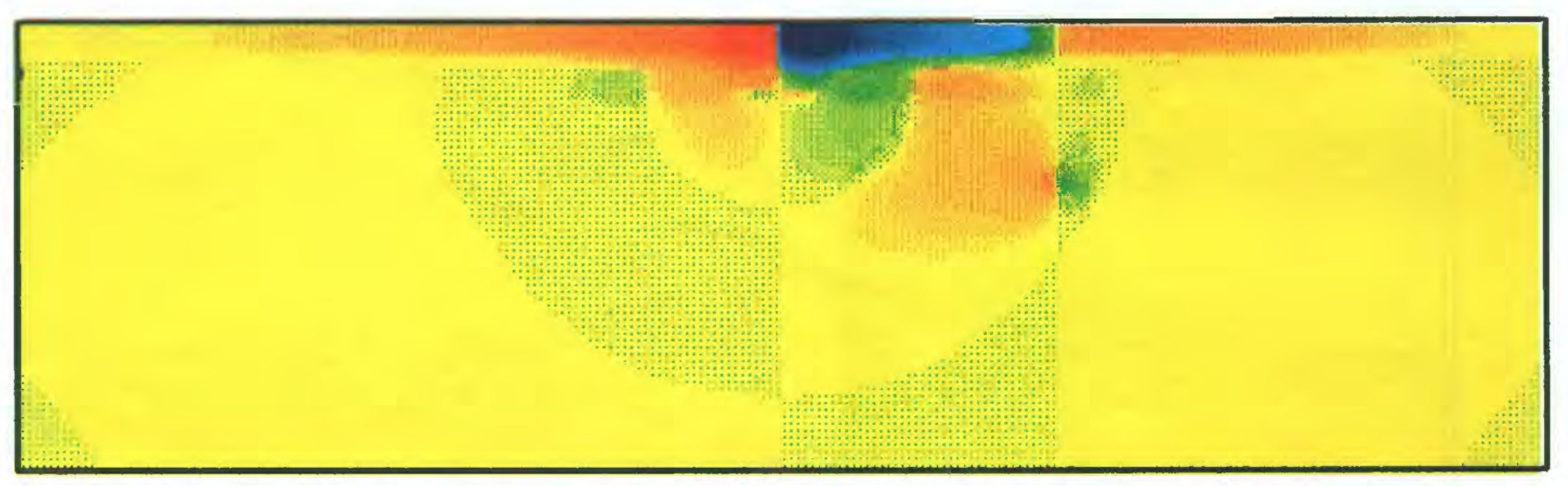

OVERBURDEN HZ CONTRIB. AT T $=.031 \mathrm{MS}$

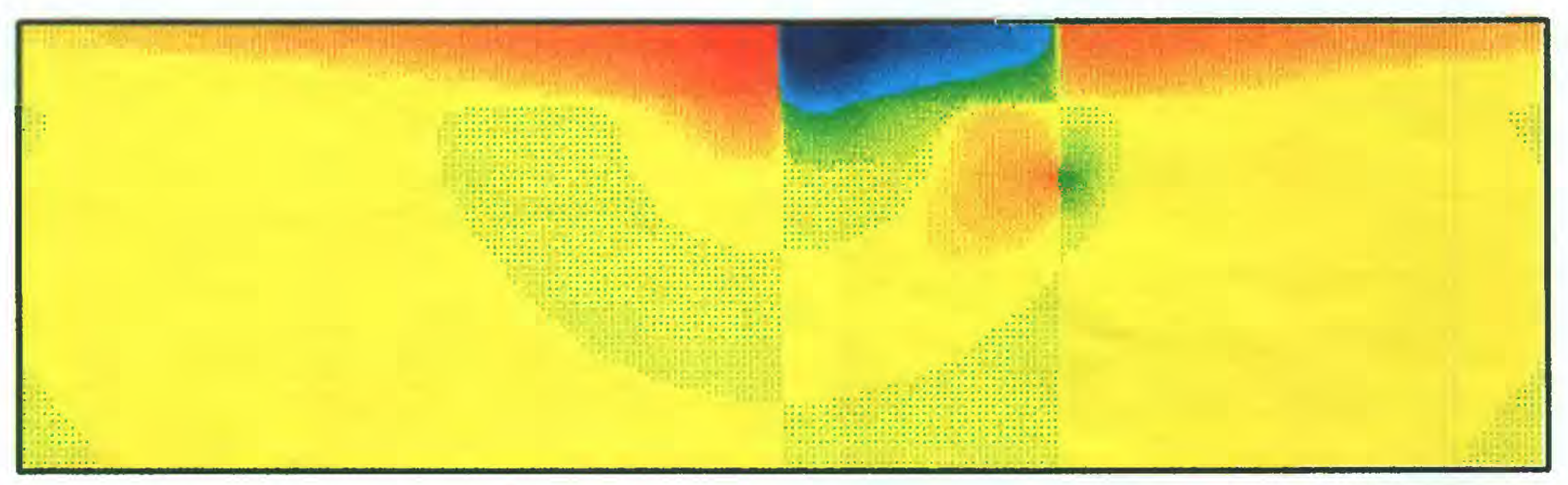

OVERBURDEN HZ CONTRIB. AT T $=.1 \mathrm{MS}$

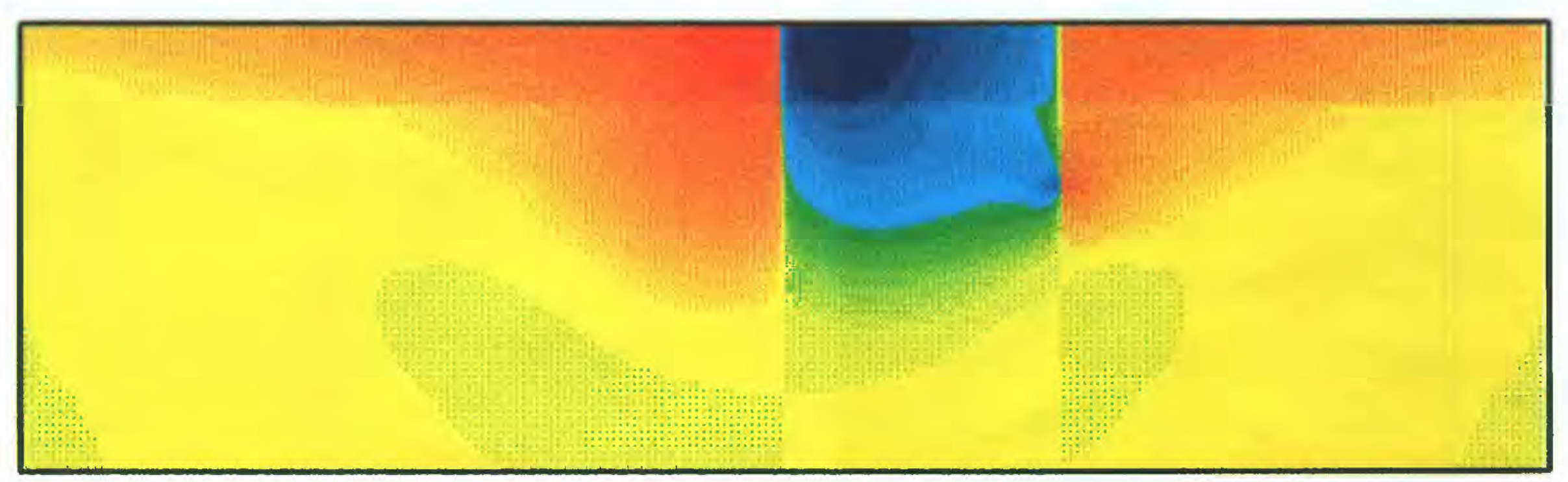

OVERBURDEN HZ CONTRIB. AT T $=.31 \mathrm{MS}$

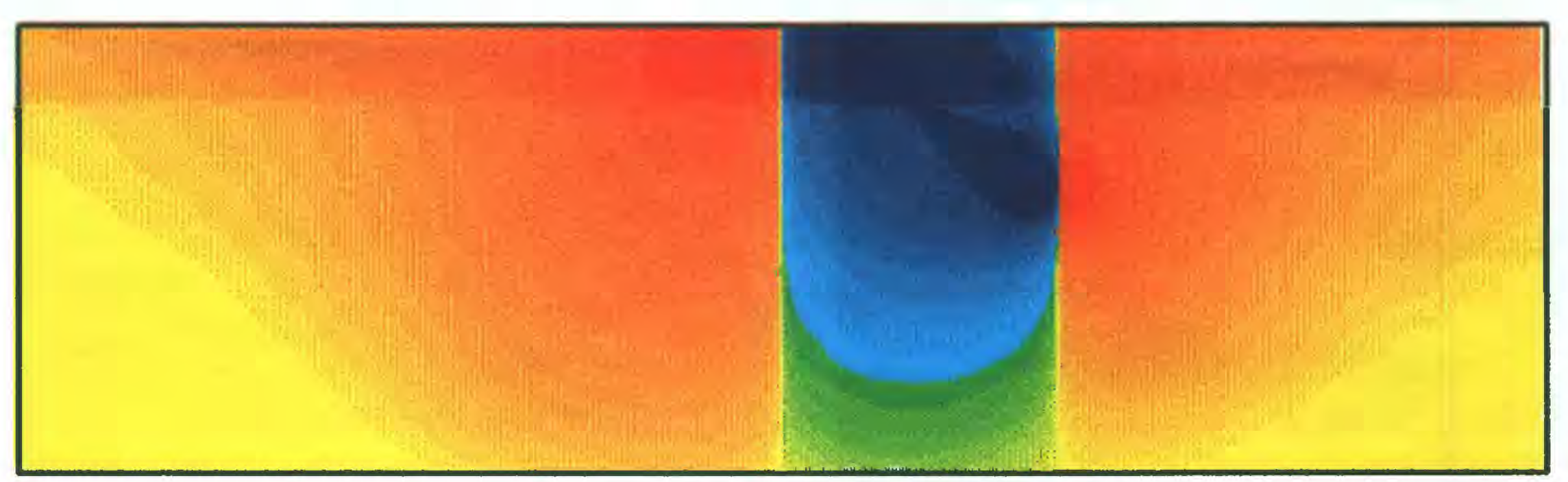


OVERBURDEN HZ CONTRIB. AT T $=1 \mathrm{MS}$

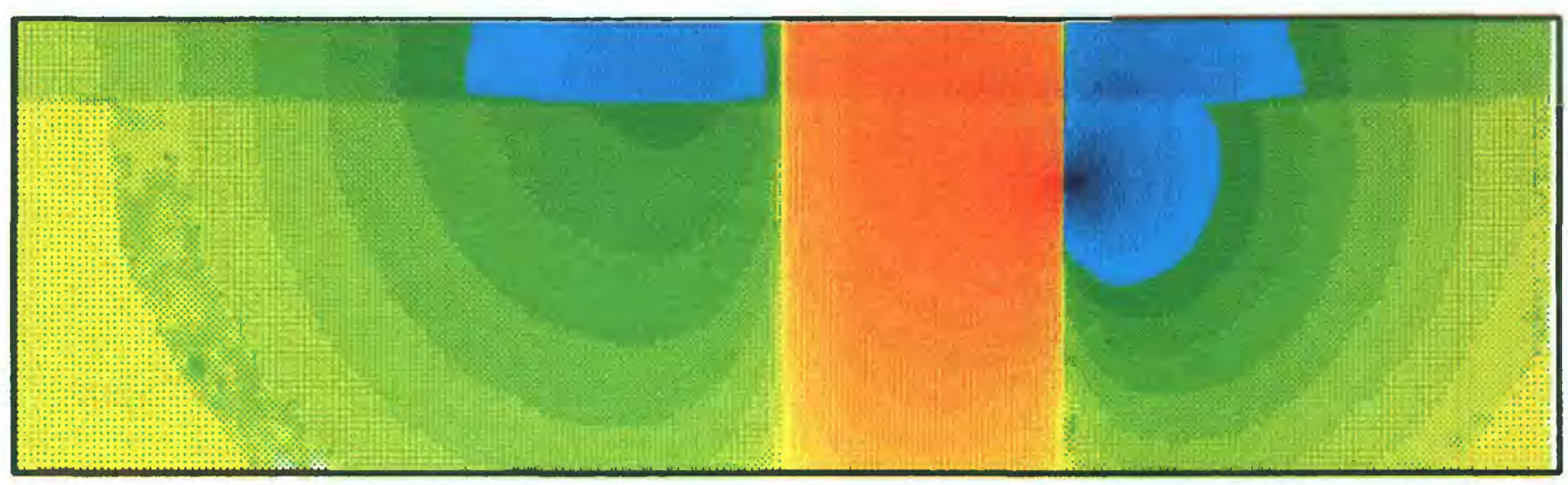

OVERBURDEN HZ CONTRIB. AT T $=3.1 \mathrm{MS}$

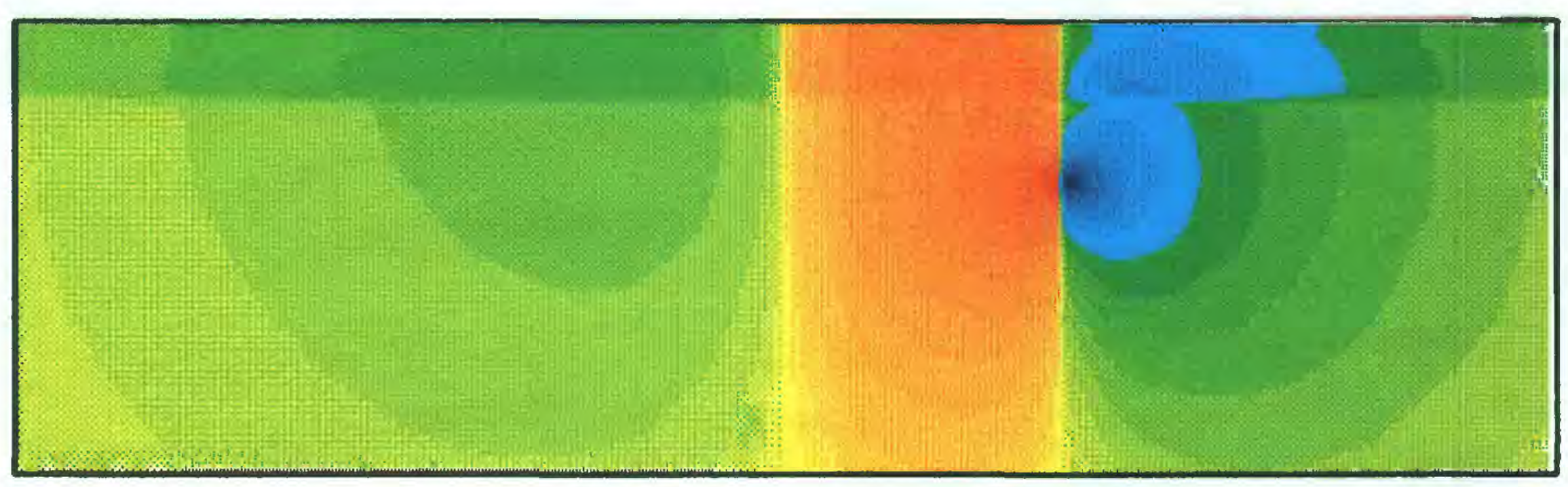

OVERBURDEN HZ CONTRIB. AT $T=10 \mathrm{MS}$

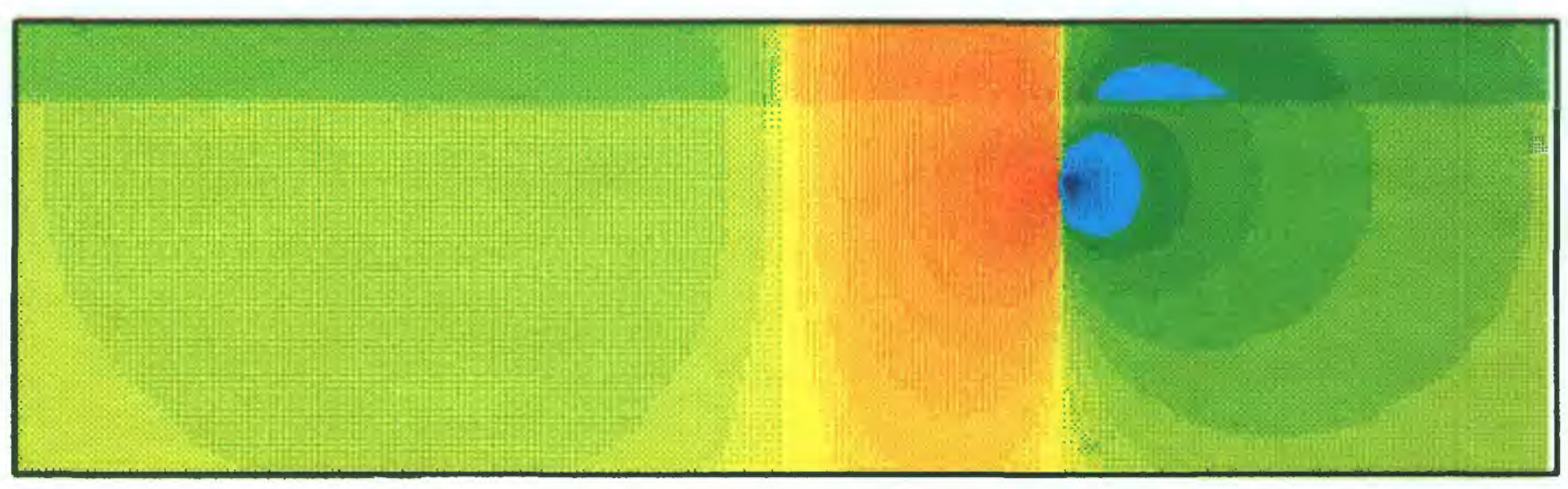

OVERBURDEN HZ CONTRIB. AT T $=31 \mathrm{MS}$

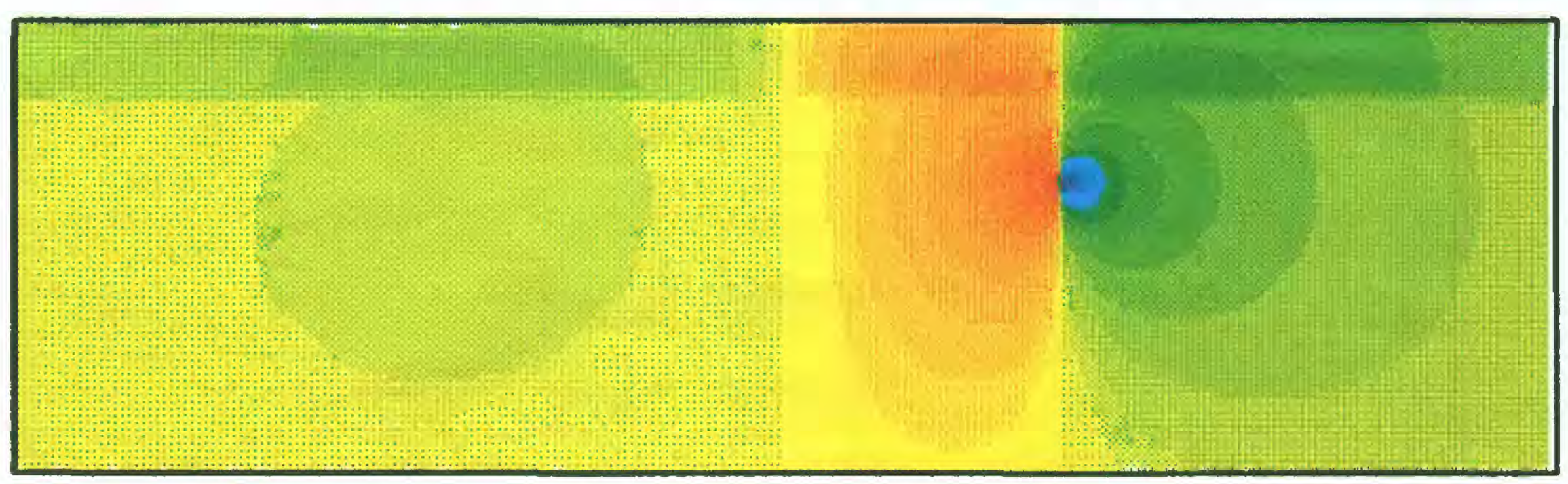

